\title{
Metallicity and distance of NGC 6362 from its RR Lyrae and SX Phoenicis stars ${ }^{\star}$
}

\author{
A. Arellano Ferro ${ }^{1, \star \star}$ J. A. Ahumada ${ }^{2}$, I.H. Bustos Fierro ${ }^{2}$, J. H. Calderónn ${ }^{2,3}$ and N. I. Morrell ${ }^{4}$ \\ 1 Instituto de Astronomía, Universidad Nacional Autónoma de México, Ciudad de México, CP 04510, Mexico \\ 2 Observatorio Astronómico, Universidad Nacional de Córdoba, Laprida 854, 5000 Córdoba, Argentina. \\ 3 Consejo Nacional de Investigaciones Científicas y Técnicas (CONYCET), Argentina. \\ ${ }^{4}$ Las Campanas Observatory, Carnegie Observatories, Casilla 601, La Serena, Chile
}

Received XXXX, accepted XXXX

Published online XXXX

Key words globular clusters: individual (NGC 6362) - Horizontal branch - RR Lyrae stars - Blue Stragglers

\begin{abstract}
New time-series VI CCD photometry of the globular cluster NGC 6362 is studied with the aim of estimating the reddening, mean metallicity and distance of the cluster from its population of RR Lyrae stars. The Fourier decomposition of carefully selected single-mode RR Lyrae light curves, and the use of well-established semi-empirical calibrations and revised zero points, lead to the values of $[\mathrm{Fe} / \mathrm{H}]_{U V E S}-1.066 \pm 0.126$ and $-1.08 \pm 0.16$ and the distance $7.93 \pm 0.32$ and $8.02 \pm 0.15$ kpc from the RRab and RRc stars respectively. The distribution of RR Lyrae stars in the horizontal branch shows a neat segregation of pulsating modes about the red edge of the first overtone instability strip, which is not necessarily expected in an OoI type cluster like NGC 6362. Four RRab stars are found likely advanced in their evolution towards the AGB. One new foreground SX Phe star, some $4 \mathrm{kpc}$ in front of the cluster and projected onto the field of our images is reported. We comment on the heavy light contamination, by a very close neighbouring star, of the peculiar double-mode V37 variable, recently postulated as a non-typical RRc variable.
\end{abstract}

\section{Introduction}

The southern globular cluster NGC 6362 (C1726-670 in the IAU nomenclature), located at $\alpha=17^{\mathrm{h}} 31^{\mathrm{m}} 55.0^{\mathrm{s}}$, $\delta=-67^{\circ} 02^{\prime} 52^{\prime \prime}(\mathrm{J} 2000), l=325.55, b=-17.57$, is at the relatively low distance from the Sun of $7.6 \mathrm{kpc}$, has a low reddening $E(B-V)=0.09$, and has a low concentration $\left(c=1.09, \rho_{0}=2.29\right)$ (Harris 1996). It is also worth mentioning that recently Dalessandro et al. (2014), based on HST observations, have identified two different, spatially mixed populations in the cluster, apparent in the subgiant and red giant branches in the $U$ vs. $(U-B)$ diagram (their Fig. 4). Dalessandro et al. (2014) also claim that NGC 6362 is one of the least massive globulars $\left(M_{\text {tot }} \sim 5 \times 10^{4} M_{\odot}\right)$ where multiple populations have been detected so far.

Given its distance, the cluster is bright, with a horizontal branch at $V \sim 15 \mathrm{mag}$, and consequently the discovery of its variable stars began early. The first 15 variables were found by Woods \& Bailey (1919) on plates taken with the 13-inch Boyden telescope at Arequipa, Peru. Much later, van Agt (1961) published the discovery of variables V16 to V31 on plates obtained by P. Th. Oosterhoff from South

\footnotetext{
* Data obtained at the CASLEO, Las Campanas and Bosque Alegre Observatories. Complejo Astronómico El Leoncito (CASLEO) is operated under agreement between the Consejo Nacional de Investigaciones Científicas y Técnicas de la República Argentina, and the National Universities of La Plata, Córdoba, and San Juan, Argentina.

$\star \star$ Corresponding author: armando@astro.unam.mx
}

Africa in 1950 with the 74-inch Radcliffe reflector at Pretoria. van Agt (1961) provided $(x, y)$ coordinates and an ID chart for all V1-V31 variables. Fourcade et al. (1966) found star V32 on plates taken with the 60-inch telescope at Bosque Alegre Astrophysical Station, Córdoba, Argentina, again giving coordinates and charts for all the variables discovered so far.van Hoof (1961) discovered V33 (his VH 11) and another seven variables that had already been independently announced by van Agt (1961). Sawyer Hogg (1973) in her 3rd catalogue adopted van Agt's numbering system, although all the epochs, periods, and magnitudes listed for the NGC 6362 variables in that catalogue are from van Hoof's paper. Much more recently, already in the CCD era, Mazur et al.(1999) discovered variables V34-52 on images taken between 1991 and 1996 with the $2.5-\mathrm{m}$ (du Pont) and 1-m (Swope) telescopes at Las Campanas, Chile, and gave $\mathrm{AR}$ and Dec coordinates with individual finding charts. The non-membership status of the eclipsing EC variables V43, V45, and V52 was confirmed by Ruciński (2000). In the Catalogue of Variable Stars in Globular Clusters (CVSGC; Clement et al. 2001), the periods, magnitudes, amplitudes, and classifications for V1-37 are from Olech et al. (2001), and for V38-52 are from Mazur et al. (1999), while the RA and Dec for most of V1-52 are from Samus et al. (2009), with the exception of stars V11, 23, 24, 26, 28, 29, 32, and 38-41 which are from Mazur et al. (1999). Finally, Kałużny et al. (2014) reported a search for variable stars carried out 
between 1995 and 2009 in the field of NGC 6362 also with the du Pont and Swope telescopes at Las Campanas; they found 25 newly detected variable stars (V53-77), including 18 proper-motion cluster members. Kałużny et al. (2014) provide identification charts and RA and Dec coordinates, although M. Rozyczka (private comm.) pointed out that the coordinates of V42, 45, 49, 53-58, 60, 63, and 75-77 are incorrect in Table 1 of their paper. A new set of high quality data obtained between 1999 and 2009 was recently employed by Smolec et al. (2017) (hereinafter Smo17), to discuss the Blazhko and double mode nature of the sample of RRab and RRc stars. With the observations reported in the present work, there is a time base of almost a century of variable star studies in NGC 6362.

As in most of our recent papers we have employed the DanDIA ${ }^{1}$ implementation of difference image analysis (DIA) (Bramich 2008) to extract high-precision photometry for all of the point sources in the field of NGC 6362. We collected 12245 light curves in the $V$ and $I$ bandpasses with the aim of building up a colour-magnitude diagram (CMD) and discussing the horizontal branch (HB) structure as compared to other Oosterhoff type I (OoI) and Oosterhoff type II (OoII) clusters ${ }^{2}$ We also Fourier decomposed the light curves of the RR Lyrae stars (RRL) to calculate their metallicity and luminosity in order to provide independent and homogeneous estimates of the cluster mean metallicity and distance.

The scheme of the paper is as follows: In $\S 2$ we describe the observations, data reduction and calibration to the standard system. In $\S 3$ the periods and phased light curves of RRL stars are displayed and the Fourier light curve decomposition of stable RRL is described and the reddening is estimated. The corresponding individual values of $[\mathrm{Fe} / \mathrm{H}]$ and $M_{V}$ are reported. $\S 4$ summarizes some properties of the SX Phe population in the cluster and reports a newly found foreground SX Phe variable. In $\S 5$ we report the distance to the cluster obtained from the Fourier decomposition of RRL stars light curves and the P-L relation of the SX Phe stars. $\S$ 6 deals with the discussion of the distribution of RRL in the HB. $\S 7$ offers a summary of the results. Appendix A gives the discussion of a few peculiar stars and in Appendix B we display a detailed finding chart of all variables in the field of NGC 6362.

\section{Observations}

The observations of this cluster were performed in three sites. First, the Complejo Astronomico El Leoncito (CASLEO), San Juan, Argentina, where the 2.15-m telescope was used on March 20-22, 2013. The detector was

\footnotetext{
1 DanDIA is built from the DanIDL library of IDL routines available at http://www.danidl.co.uk.

2 Oosterhoff (1939) showed that globular clusters can be distinguished according to the mean perdiod of their fundamental mode RR Lyrae stars, or RRab, being $\sim 0.55$ days in OoI and $\sim 0.65$ days in OoII systems, and noted that the percentage of first overtone RR Lyrae stars, or RRc, is lower in OoI than in OoII clusters.
}
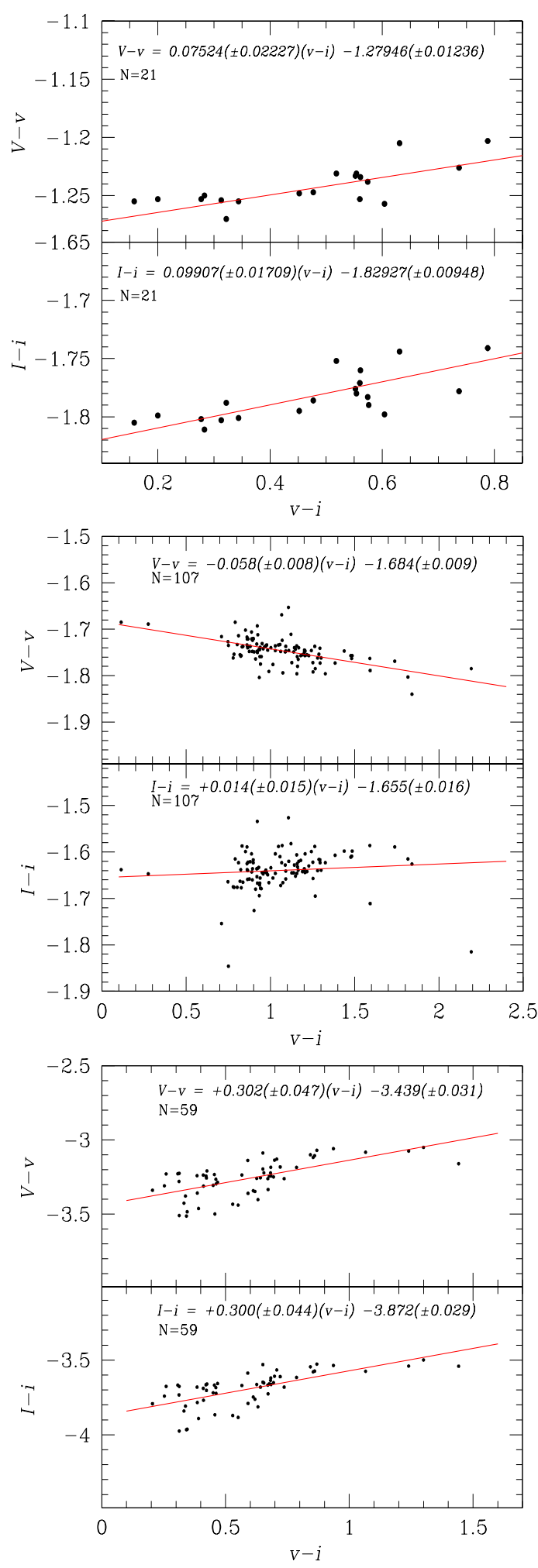

Fig. 1 The transformation relation for $V$ and $I$ for the three sets of data: from top to bottom; CASLEO, SWOPE and Bosque Alegre. Note that data from Bosque Alegre have the largest colour dependence. The transformation equations are given in each panel. 
a Roper Scientific back-illuminated CCD of $2048 \times 2048$ pixels with a scale of $0.15 \mathrm{arcsec} / \mathrm{pix}$ and a field of view (FoV) of approximately $5.1 \times 5.1 \mathrm{arcmin}^{2}$. Second,the $1-\mathrm{m}$ Swope Telescope of the Las Campanas Observatory was employed on June 3 and 14, 2014, and the E2V CCD231-84 of 4096x4112 pixels with a scale of $0.435 \mathrm{arcsec} /$ pix and a FoV of approximately $14.5 \times 14.5 \operatorname{arcmin}^{2}$. Finally, on June 20, August 21 and 22 and September 5 and 6, 2015, we used the 1.54-m telescope of the Bosque Alegre Observatory, Cordoba, Argentina equipped with a CCD Alta U9 of $3072 \times 2048$ pixels, with a scale of $0.247 \mathrm{arcsec} / \mathrm{pix}$ and a FoV of approximately $12.6 \times 8.4 \mathrm{arcmin}^{2}$. The log of our observations is given in Table 1 .

\subsection{Difference image analysis}

As in previous papers we employed the difference image analysis (DIA) technique and the DanDIA pipeline (Bramich (2008); Bramich et al. (2013)) to extract highprecision photometry of all point sources in the images of NGC 6362. The procedure and its caveats have been described in detail by Bramich et al. (2011).

\subsection{Photometric calibrations}

\subsubsection{Relative calibration}

Systematic errors in photometric data may be so severe that they mimic bona fide stellar variability. Time-series photometry of a set of non-variable objects may be used to calculate and correct these systematic errors. We have applied the methodology developed by Bramich \& Freudling (2012) to solve for magnitude offsets $Z_{k}$ that should be applied to each photometric measurement from image $k$.

\subsubsection{Absolute calibration}

The transformation to the standard VI system was performed using the local standards in the FoV from the collection of Stetson 2000 The calibrations for the three telescopes are shown in Fig. 1. The transformation equations to the standard $V$ and $I$ system for each data set are given in the corresponding panel. In Table 2 we report the $V$ and $I$ photometry for all RRL stars inour FoV. The full table is published in electronic format, although we include a small portion of it the printed version.

\section{The RR Lyrae stars}

The resulting light curves of the RR Lyrae stars combining the observations from the runs in the three sites are shown in Figs. 2 and 3 . The zero points for CASLEO and LC match very well. For BA however we found small drifts that vary from star to star. With the aim of using all data to refine

3 http://www.cadc-ccda.hia-iha.nrc-cnrc.gc.ca/en/community/ STETSON/standards/
Table 1 Observations log of NGC 6362. Data are from three sites; CASLEO (CAS), Las Campanas (LC) and Bosque Alegre (BA). Columns $N_{V}$ and $N_{I}$ give the number of images taken with the $V$ and $I$ filters respectively. Columns $t_{V}$ and $t_{I}$ provide the exposure time, or range of exposure times. In the last column the average seeing is listed.

\begin{tabular}{lcccccc}
\hline Date & Site & $N_{V}$ & $t_{V}(\mathrm{~s})$ & $N_{I}$ & $t_{I}(\mathrm{~s})$ & seeing (") \\
\hline 20130321 & CAS & 15 & 300 & 9 & 240 & 2.7 \\
20130322 & CAS & 8 & 480 & 9 & 220 & 2.7 \\
20130323 & CAS & 8 & 360 & 11 & 240 & 2.0 \\
20140504 & CAS & 22 & 480 & 30 & $180-220$ & 3.1 \\
20140605 & LC & 140 & $4-20$ & 79 & $2-10$ & 2.1 \\
20140615 & LC & 88 & $4-20$ & 53 & $2-10$ & 1.7 \\
20150621 & BA & 37 & $180-300$ & 36 & $120-180$ & 1.8 \\
20150821 & BA & 8 & 300 & 11 & 150 & 3.5 \\
20150821 & BA & 11 & 300 & 14 & 150 & 2.7 \\
20150906 & BA & 27 & 300 & 27 & 150 & 2.5 \\
20150907 & BA & 3 & 300 & 6 & 150 & 1.9 \\
\hline Total: & & 367 & - & 285 & - & - \\
\hline
\end{tabular}

the period, we applied these drifts and then the light curves were phased with the new resulting periods listed in column 9 of Table 3 We also report itensity-weighted mean $V$ and $I$ magnitudes, amplitudes and equatorial coordinates for all the RR Lyrae stars. For comparison we also include the periods recently reported by Smo17.

\subsection{The reddening of NGC 6362 from its RRab stars}

Using the fact that RRab stars have nearly the same intrinsic colour $(B-V)_{0}$ at minimum light (Sturch 1966), one can calculate the individual reddenings of these stars, which can provide a good average of the cluster reddening or to reveal the presence of differential reddening. The $(V-I)_{0}$ at minimum has been calibrated by Guldenschuh et al. (2005) as $(V-I)_{0, \min }=0.58 \pm 0.02$. We have adopted this value and the minimum $(V-I)$ from our observations to estimate $E(V-I)$ for each RRab with a well defined minimum. This was converted to $E(B-V)$ through the ratio $E(V-I) / E(B-V)=1.259$ derived from Schlegel et al. (1998). From 15 RRab stars we find an average of $E(B-V)=0.063 \pm 0.024$. The scatter is small and shows no signs of differential reddening, hence, we shall adopt this value for the determination of the distance to NGC 6362 via the several approaches described in $\S 5$. The above reddening calculation can be compared with previous estimations such as 0.10 (Olech et al. 2001), 0.06 \pm 0.03 (Piotto et al. 1999) and 0.08 (Brocato et al.|1999).

\subsection{Fourier decomposition and physical parameters of RR Lyrae stars}

Determination of $[\mathrm{Fe} / \mathrm{H}]$ and $M_{V}$ of RR Lyrae stars in a given cluster enables the estimation of the mean values of the metallicity and distance of the parental cluster. This can be achieved via the Fourier decomposition of their light curves and the employment of well established calibrations and their zero points of these physical quantities and the 

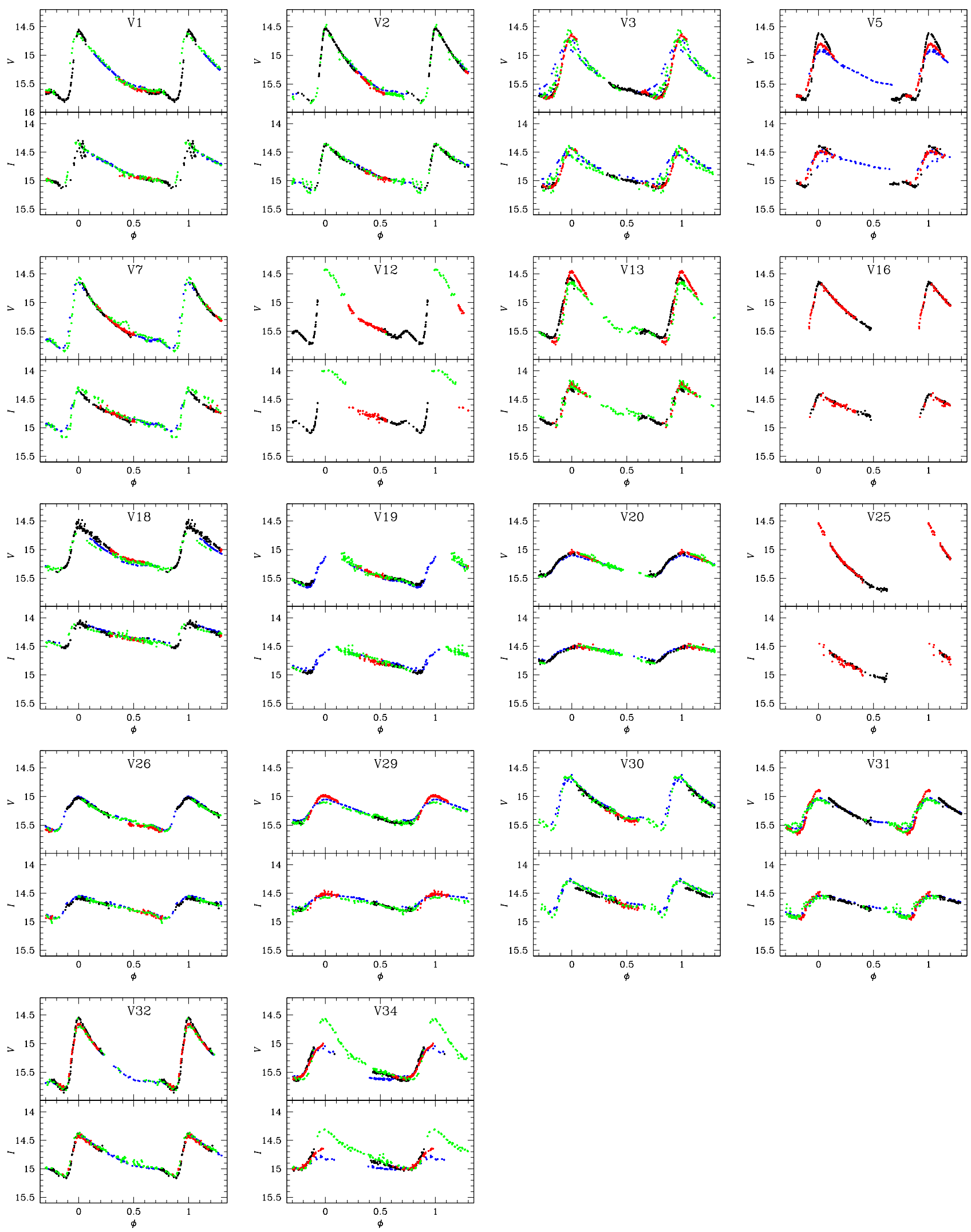

Fig. 2 Light curves of RRab stars. Colours are assigned as per observatory as follows: blue for CASLEO, red and black for Las Campanas and green for Bosque Alegre. 
Table 2 Time-series $V$ and $I$ photometry for all RR Lyrae stars in our field of view. The standard $M_{\text {std }}$ and instrumental $m_{\text {ins }}$ magnitudes are listed in columns 4 and 5, respectively, corresponding to the variable stars in column 1. Filter and epoch of mid-exposure (in heliocentric julian days) are listed in columns 2 and 3, respectively. The uncertainty on $m_{\text {ins }}$ is listed in column 6, which also corresponds to the uncertainty on $M_{\mathrm{std}}$. For completeness, we also list the reference and differential fluxes $f_{\text {dref }}$ and $f_{\text {diff }}$ and the scale factor $p$ in columns 7,9, and 11, along with the uncertainties $\sigma_{\text {ref }}$ and $\sigma_{\text {diff }}$ in columns 8 and 10. This is an extract from the full table, which is available with the electronic version of the article.

\begin{tabular}{ccccccccccc}
\hline $\begin{array}{c}\text { Variable } \\
\text { Star ID }\end{array}$ & Filter & $\begin{array}{c}\text { HJD } \\
(\mathrm{d})\end{array}$ & $\begin{array}{c}M_{\text {std }} \\
(\mathrm{mag})\end{array}$ & $\begin{array}{c}m_{\text {ins }} \\
(\mathrm{mag})\end{array}$ & $\begin{array}{c}\sigma_{m} \\
(\mathrm{mag})\end{array}$ & $\begin{array}{c}f_{\text {ref }} \\
\left(\mathrm{ADU} \mathrm{s}^{-1}\right)\end{array}$ & $\begin{array}{c}\sigma_{\text {ref }} \\
\left(\mathrm{ADU} \mathrm{s}^{-1}\right)\end{array}$ & $\begin{array}{c}f_{\text {diff }} \\
\left(\mathrm{ADU} \mathrm{s}^{-1}\right)\end{array}$ & $\begin{array}{c}\sigma_{\text {diff }} \\
\left(\mathrm{ADU} \mathrm{s}^{-1}\right)\end{array}$ & $p$ \\
\hline V1 & $V$ & 2456372.79007 & 14.994 & 16.275 & 0.001 & 631.204 & 1.835 & +536.163 & 3.076 & 3.7921 \\
V1 & $V$ & 2456372.79452 & 15.015 & 16.296 & 0.001 & 631.204 & 1.835 & +481.905 & 2.989 & 3.8046 \\
$\vdots$ & $\vdots$ & $\vdots$ & $\vdots$ & $\vdots$ & $\vdots$ & $\vdots$ & $\vdots$ & $\vdots$ & $\vdots$ \\
V1 & $I$ & 2456372.78347 & 14.559 & 16.390 & 0.003 & 1406.814 & 3.984 & -33.805 & 7.146 & 1.9506 \\
V1 & $I$ & 2456372.80310 & 14.602 & 16.434 & 0.002 & 1406.814 & 3.984 & -140.873 & 3.975 & 1.9684 \\
$\vdots$ & $\vdots$ & $\vdots$ & $\vdots$ & $\vdots$ & $\vdots$ & $\vdots$ & $\vdots$ & $\vdots$ & $\vdots$ & 3.956 \\
V2 & $V$ & 2456372.79007 & 15.040 & 16.317 & 0.001 & 489.291 & 1.760 & +963.456 & 3.7921 \\
V2 & $V$ & 2456372.79452 & 15.060 & 16.337 & 0.001 & 489.291 & 1.760 & +914.472 & 2.881 & 3.8046 \\
$\vdots$ & $\vdots$ & $\vdots$ & $\vdots$ & $\vdots$ & $\vdots$ & $\vdots$ & $\vdots$ & $\vdots$ & $\vdots$ \\
V2 & $I$ & 2456372.78347 & 14.583 & 16.409 & 0.003 & 1194.571 & 4.129 & +333.684 & 6.998 & 1.9506 \\
V2 & $I$ & 2456372.80310 & 14.639 & 16.466 & 0.002 & 1194.571 & 4.129 & +200.011 & 3.851 & 1.9684 \\
$\vdots$ & $\vdots$ & $\vdots$ & $\vdots$ & $\vdots$ & $\vdots$ & $\vdots$ & $\vdots$ & $\vdots$ & $\vdots$ \\
\hline
\end{tabular}

corresponding Fourier parameters. By doing it on a homogeneous basis, i.e. using the same semi-empirical calibrations and zero points for a family of globular clusters of both Oosterhoff types, OoI and OoII, an independent insight on the metallicity dependence of the horizontal branch (HB) luminosity, i.e., the familiar $M_{V^{-}}[\mathrm{Fe} / \mathrm{H}]$ relation can be obtained. Such approach has been applied by Arellano Ferro et al. (2017) to a group of 23 globular clusters. While preliminary results for NGC 6362 were included in that paper, in the present work we publish the specific values of the Fourier parameters and the individual physical parameters for a carefully selected sample of stable RRab stars and RRc stars in the cluster, according to the accurate photometry of Smo17.

Although the procedure and the employed calibrations have been described in detail by Arellano Ferro et al. (2017) and in several papers cited there, for completeness we include here the fundamentals.

The form of the Fourier representation of a given light curve is:

$m(t)=A_{0}+\sum_{k=1}^{N} A_{k} \cos \left(\frac{2 \pi}{P} k(t-E)+\phi_{k}\right)$,

where $m(t)$ is the magnitude at time $t, P$ is the period, and $E$ is the epoch. A linear minimization routine is used to derive the best fit values of the amplitudes $A_{k}$ and phases $\phi_{k}$ of the sinusoidal components. From the amplitudes and phases of the harmonics in Eq. 11, the Fourier parameters, defined as $\phi_{i j}=j \phi_{i}-i \phi_{j}$, and $R_{i j}=A_{i} / A_{j}$, are computed.

Since some of the calibrations and zero points employed in this work towards the calculation of stellar physical quantities differ from the ones used in the previous Fourier decomposition analysis for the RR Lyrae stars in NGC 6362
Olech et al. (2001), below we explicitly list the calibrations we used:

$[\mathrm{Fe} / \mathrm{H}]_{J}=-5.038-5.394 P+1.345 \phi_{31}^{(s)}$,

$M_{V}=-1.876 \log P-1.158 A_{1}+0.821 A_{3}+K$,

given by Jurcsik \& Kovacs (1996) and Kovács \& Walker (2001), respectively. The standard deviations of the above calibrations are $0.14 \mathrm{dex}$ (Jurcsik 1998) and $0.04 \mathrm{mag}$, respectively. In eq. 3 we have used $\mathrm{K}=0.41$ (see the discussion in Section 4.2 of Arellano Ferro et al. (2010)). Eq. 2 is applicable to RRab stars with a deviation parameter $D_{m}$, defined by Jurcsik \& Kovacs (1996) and Kovacs \& Kanbur (1998), not exceeding an upper limit. These authors suggest $D_{m} \leq 3.0$. The $D_{m}$ is listed in column 11 of Table 4, where it is obvious that the five stable RRab stars have light curves consistent with the calibration of eq. 2 .

For the RRc stars we employ the calibrations:

$$
\begin{gathered}
{[\mathrm{Fe} / \mathrm{H}]_{Z W}=52.466 P^{2}-30.075 P+0.131 \phi_{31}^{(c)}{ }^{2}} \\
\quad-0.982 \phi_{31}^{(c)}-4.198 \phi_{31}^{(c)} P+2.424, \\
M_{V}=1.061-0.961 P-0.044 \phi_{21}^{(s)}-4.447 A_{4},
\end{gathered}
$$

given by Morgan et al. (2007) and Kovács (1998), respectively. For eq. 5 the zero point was reduced to $1.061 \mathrm{mag}$ to make the luminosities of the RRc consistent with the distance modulus of $18.5 \mathrm{mag}$ for the LMC (see discussions by Cacciari et al. (2005) and Arellano Ferro et al. (2010)). The original zero point given by Kovács (1998) is 1.261.

When necessary, the coefficients were transformed from cosine series phases into the sine series via the relation $\phi_{j k}^{(s)}=\phi_{j k}^{(c)}-(j-k) \frac{\pi}{2}$ 
Table 3 General data for the RR Lyrae stars in NGC 6362 in the FoV of our images. The variable types are adopted from the work of Smo17. Previous period estimates for each variable from Smo17 are reported in column 7 for comparison with our periods in column 9.

\begin{tabular}{|c|c|c|c|c|c|c|c|c|c|c|}
\hline $\begin{array}{l}\text { Variable } \\
\text { Star ID }\end{array}$ & $\begin{array}{l}\text { Variable } \\
\text { Type }\end{array}$ & $\begin{array}{l}<V> \\
(\mathrm{mag})\end{array}$ & $\begin{array}{l}<I> \\
(\mathrm{mag})\end{array}$ & $\begin{array}{l}A_{V} \\
\text { (mag) }\end{array}$ & $\begin{array}{l}A_{I} \\
(\mathrm{mag})\end{array}$ & $\begin{array}{l}P(\text { Smo17) } \\
\text { (d) }\end{array}$ & $\begin{array}{l}\mathrm{HJD}_{\max } \\
(+2450000)\end{array}$ & $\begin{array}{l}P \text { (this work) } \\
\text { (d) }\end{array}$ & $\begin{array}{l}\text { RA } \\
\text { (J2000.0) }\end{array}$ & $\begin{array}{l}\text { Dec } \\
(\text { J2000.0) }\end{array}$ \\
\hline V1 & RRab $B l$ & 15.352 & 14.804 & 1.292 & 0.782 & 0.50479162 & 6813.9219 & 0.504814 & $17: 31: 54.72$ & $-67: 02: 45.7$ \\
\hline $\mathrm{V} 2$ & RRab & 15.368 & 14.834 & 1.366 & 0.856 & 0.48897301 & 6813.7537 & 0.488972 & $17: 31: 50.23$ & $-67: 04: 25.4$ \\
\hline V3 & $\mathrm{RRd}$ & 15.378 & 14.866 & 1.185 & 0.774 & 0.44728792 & 6823.8616 & 0.447297 & $17: 31: 40.91$ & $-67: 04: 15.8$ \\
\hline V5 & RRab $B l$ & 15.348 & 14.761 & 1.177 & 0.716 & 0.52083783 & 6813.8986 & 0.521434 & $17: 32: 08.82$ & $-67: 02: 59.4$ \\
\hline V6 & RRc $B l$ & 15.312 & 14.911 & 0.47 & 0.28 & 0.26270671 & 6813.8662 & 0.238084 & $17: 32: 03.85$ & $-66: 59: 51.8$ \\
\hline V7 & RRab $B l$ & 15.368 & 14.783 & 1.242 & 0.872 & 0.521581388 & 6781.8912 & 0.521572 & $17: 31: 58.52$ & $-67: 01: 01.4$ \\
\hline V8 & RRc $n r$ & 15.082 & 14.620 & 0.517 & 0.321 & 0.38148471 & 7194.7619 & 0.381466 & $17: 31: 10.05$ & $-67: 01: 01.3$ \\
\hline V10 & RRc $B l$ & 15.257 & 14.970 & 0.471 & 0.254 & 0.265638816 & 6813.8947 & 0.315140 & $17: 32: 26.13$ & $-66: 56: 52.6$ \\
\hline V11 & $\mathrm{RRc}$ & 15.255 & 14.869 & 0.502 & 0.317 & 0.288789268 & 6823.7797 & 0.288789 & $17: 31: 49.90$ & $-67: 01: 57.8$ \\
\hline V12 & RRab $B l$ & 15.227 & 14.641 & 1.308 & 1.103 & 0.5328814 & 7194.6017 & 0.533046 & $17: 31: 13.13$ & $-67: 04: 30.9$ \\
\hline V13 & RRab $B l$ & 15.258 & 14.651 & 1.251 & 0.741 & 0.58002740 & 6823.8027 & 0.580010 & $17: 31: 15.14$ & $-67: 04: 47.6$ \\
\hline V14 & $\mathrm{RRc}$ & 15.374 & 15.056 & 0.344 & 0.213 & 0.24620647 & 6823.7091 & 0.252368 & $17: 32: 57.94$ & $-67: 02: 12.8$ \\
\hline V15 & RRc $n r$ & 15.263 & 14.841 & 0.448 & 0.290 & 0.279945707 & 6823.7574 & 0.279944 & $17: 32: 03.47$ & $-67: 02: 44.3$ \\
\hline V16 & $\mathrm{RRab}$ & 15.359 & 14.774 & 1.12 & 0.67 & 0.525674215 & 6813.7472 & 0.525711 & $17: 31: 58.10$ & $-67: 07: 12.0$ \\
\hline V17 & RRc $n r$ & 15.335 & 14.861 & 0.511 & 0.265 & 0.31460473 & 6813.8251 & 0.314604 & $17: 32: 29.46$ & $-67: 03: 51.2$ \\
\hline V18 & RRab $B l$ & 15.073 & 14.320 & 0.913 & 0.461 & 0.51288484 & 6813.7955 & 0.512899 & $17: 32: 13.59$ & $-67: 01: 33.1$ \\
\hline V19 & RRab & 15.365 & 14.730 & 0.663 & 0.480 & 0.59450528 & 6781.9170 & 0.594511 & $17: 32: 15.96$ & $-67: 03: 09.4$ \\
\hline V20 & RRab $B l$ & 15.273 & 14.627 & 0.436 & 0.329 & 0.69835898 & 6823.7125 & 0.698361 & $17: 32: 02.64$ & $-67: 02: 59.4$ \\
\hline $\mathrm{V} 21$ & RRc $n r$ & 15.321 & 14.945 & 0.535 & 0.362 & 0.281390043 & 6813.7955 & 0.281392 & $17: 32: 22.59$ & $-67: 04: 30.6$ \\
\hline $\mathrm{V} 22$ & $\mathrm{RRc}$ & 15.318 & 14.939 & 0.516 & 0.335 & 0.26683523 & 6823.7091 & 0.253262 & $17: 32: 26.74$ & $-67: 07: 55.1$ \\
\hline V23 & RRc $n r$ & 15.359 & 14.980 & 0.571 & 0.345 & 0.275105063 & 6813.8251 & 0.275108 & $17: 32: 00.12$ & $-67: 03: 07.5$ \\
\hline V24 & RRc $n r$ & 15.195 & 14.795 & 0.489 & 0.240 & 0.32936190 & 6813.7257 & 0.329318 & $17: 32: 07.16$ & $-67: 03: 20.3$ \\
\hline V25 & $\mathrm{RRab}$ & 15.45 & 14.9 & 1.23 & 0.74 & 0.455890887 & 6823.6985 & 0.455824 & $17: 30: 54.37$ & $-67: 06: 18.5$ \\
\hline V26 & RRab & 15.351 & 14.750 & 0.610 & 0.403 & 0.60217449 & 6781.8739 & 0.602179 & $17: 31: 58.87$ & $-67: 03: 22.2$ \\
\hline V27 & RRc $n r$ & 15.253 & 14.957 & 0.520 & 0.303 & 0.27812399 & 6813.9520 & 0.322892 & $17: 31: 21.62$ & $-66: 56: 27.7$ \\
\hline V28 & RRc $B l ?$ & 15.111 & 14.624 & 0.457 & 0.271 & 0.3584133 & 6372.8559 & 0.358399 & $17: 31: 59.16$ & $-67: 02: 07.7$ \\
\hline V29 & RRab $B l$ & 15.272 & 14.658 & 0.502 & 0.321 & 0.64778329 & 6823.8064 & 0.647743 & $17: 31: 52.49$ & $-67: 03: 20.2$ \\
\hline V30 & RRab $B l$ & 15.186 & 14.599 & 0.954 & 0.642 & 0.61340457 & 6373.8773 & 0.613415 & $17: 31: 39.59$ & $-67: 01: 33.6$ \\
\hline V31 & RRab $B l$ & 15.323 & 14.714 & 0.749 & 0.460 & 0.60021294 & 6823.8726 & 0.600220 & $17: 31: 49.20$ & $-67: 01: 20.7$ \\
\hline V32 & RRab $B l$ & 15.385 & 14.817 & 1.258 & 0.765 & 0.49724171 & 6813.8468 & 0.497248 & $17: 32: 01.83$ & $-67: 02: 12.7$ \\
\hline V33 & $\mathrm{RRc} n r$ & 15.319 & 14.924 & 0.398 & 0.231 & 0.30641758 & 6813.7889 & 0.338096 & $17: 32: 47.91$ & $-66: 56: 36.2$ \\
\hline V34 & RRd & 15.305 & 14.779 & 1.070 & 0.708 & 0.49432939 & 7194.6212 & 0.494306 & $17: 31: 52.81$ & $-67: 03: 34.3$ \\
\hline V35 & $\mathrm{RRc} n r$ & 15.318 & 14.883 & 0.462 & 0.289 & 0.29079074 & 6813.7263 & 0.290792 & $17: 32: 08.14$ & $-67: 03: 03.0$ \\
\hline V36 & $\mathrm{RRc} B l / n r$ & 15.173 & 14.698 & 0.393 & 0.248 & 0.31009148 & 6813.7294 & 0.3101 & $17: 31: 43.57$ & $-67: 02: 16.8$ \\
\hline V37 & blRR ? & 15.319 & 14.974 & 0.403 & 0.176 & 0.25503903 & 6813.9287 & 0.254576 & $17: 31: 32.12$ & $-67: 02: 03.4$ \\
\hline
\end{tabular}

$B l$ : Blazhko modulations; $n r$ :doble mode with at least a non-radial mode according to Smo17.

The values of $A_{0}$, in $V$ and $I$, for all the RRL stars in NGC 6362 are given in Table 3 as the intensity weighted quantities $<V>$ and $<I>$. However, several of these stars have been identified as Blazhko variables or double mode pulsators (Smo17), hence we have limited the Fourier decomposition, for the purpose of physical parameters determination, to those stars proven to be stable in the time scale of the analysis of Smo17. Clearly, the light curves of Smo17 are more dense and much better covered than ours in Figs. 2 and 3, since their time-base is much longer. Hence, we decided to Fourier decompose the light curves from Smo17 for the rather stable stars (i.e. the RRc stars V11, V14, V15, V21, V22 and V23, and the RRab stars V2, V16, V19, V25 and V26). The Fourier decomposition parameters for these stars and their corresponding physical parameters are listed in Tables 4 and 5 respectively. The absolute magnitude $M_{V}$ was converted into luminosity with $\log \left(L / \mathrm{L}_{\odot}\right)=-0.4\left(M_{V}-M_{\text {bol }}^{\odot}+B C\right)$. The bolometric correction was calculated using the formula $B C=$ $0.06[\mathrm{Fe} / \mathrm{H}]_{Z W}+0.06$ given by Sandage \& Cacciari (1990). We adopted $M_{\mathrm{bol}}^{\odot}=4.75 \mathrm{mag}$. For the distance calculation, and given that there are no signs of differential reddening for NGC 6362, we have adopted $E(B-V)=0.063$ (see $\S 3.1$ The weighted average of $[\mathrm{Fe} / \mathrm{H}]$ and distance are considered good mean values for the parental cluster. We found $[\mathrm{Fe} / \mathrm{H}]_{Z W}=-1.203 \pm 0.126$ and $-1.21 \pm 0.16$ for the RRab and RRc stars respectively, which in the scale of 

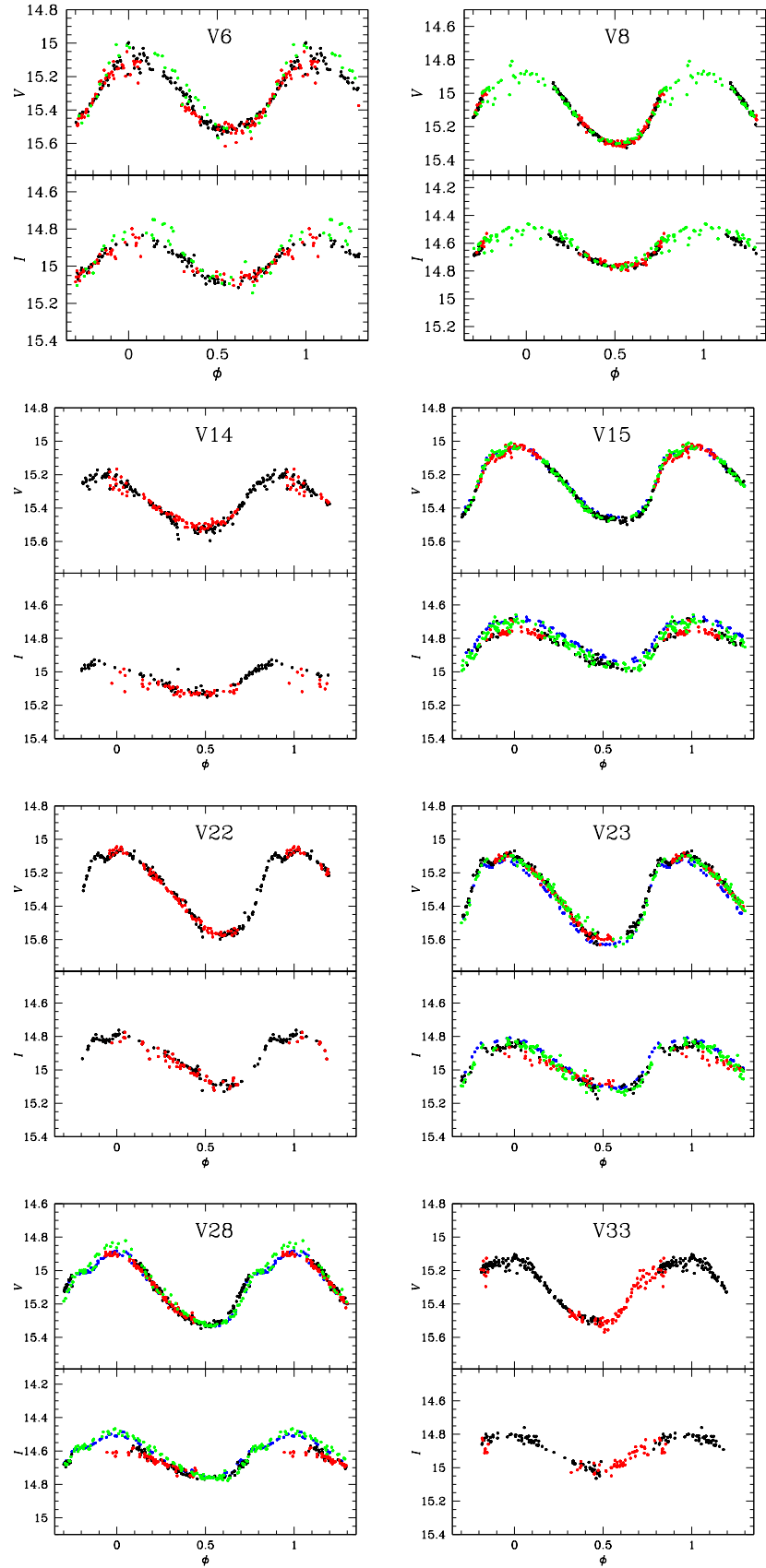

Fig. 3 Light curves of RRc stars. Colours are as in Fig. 2

Carretta et al. (2009) are $[\mathrm{Fe} / \mathrm{H}]_{U V E S}=-1.066 \pm 0.126$ and $-1.08 \pm 0.16$

The value of $[\mathrm{Fe} / \mathrm{H}]_{U V E S}$ obtained above can be compared with independent determinations of the metallicity of the cluster that can be found in the literature. The value given in the catalogue of globular cluster parameters of Harris $(1996)$ is $[\mathrm{Fe} / \mathrm{H}]_{U V E S}=-0.99$; Olech et al. (2001) finds $[\mathrm{Fe} / \mathrm{H}]_{Z W}=-1.08$ or $[\mathrm{Fe} / \mathrm{H}]_{U V E S}=-0.97$. On spectroscopic grounds Carretta \& Gratton (1997) found $[\mathrm{Fe} / \mathrm{H}]=-0.96$ from an analysis of echelle spectra of cluster giants, and Rutledge et al. (1997) found [Fe/H] $=-0.99 \pm$ 0.03 from Ca II triplet index. More recent spectroscopic de-
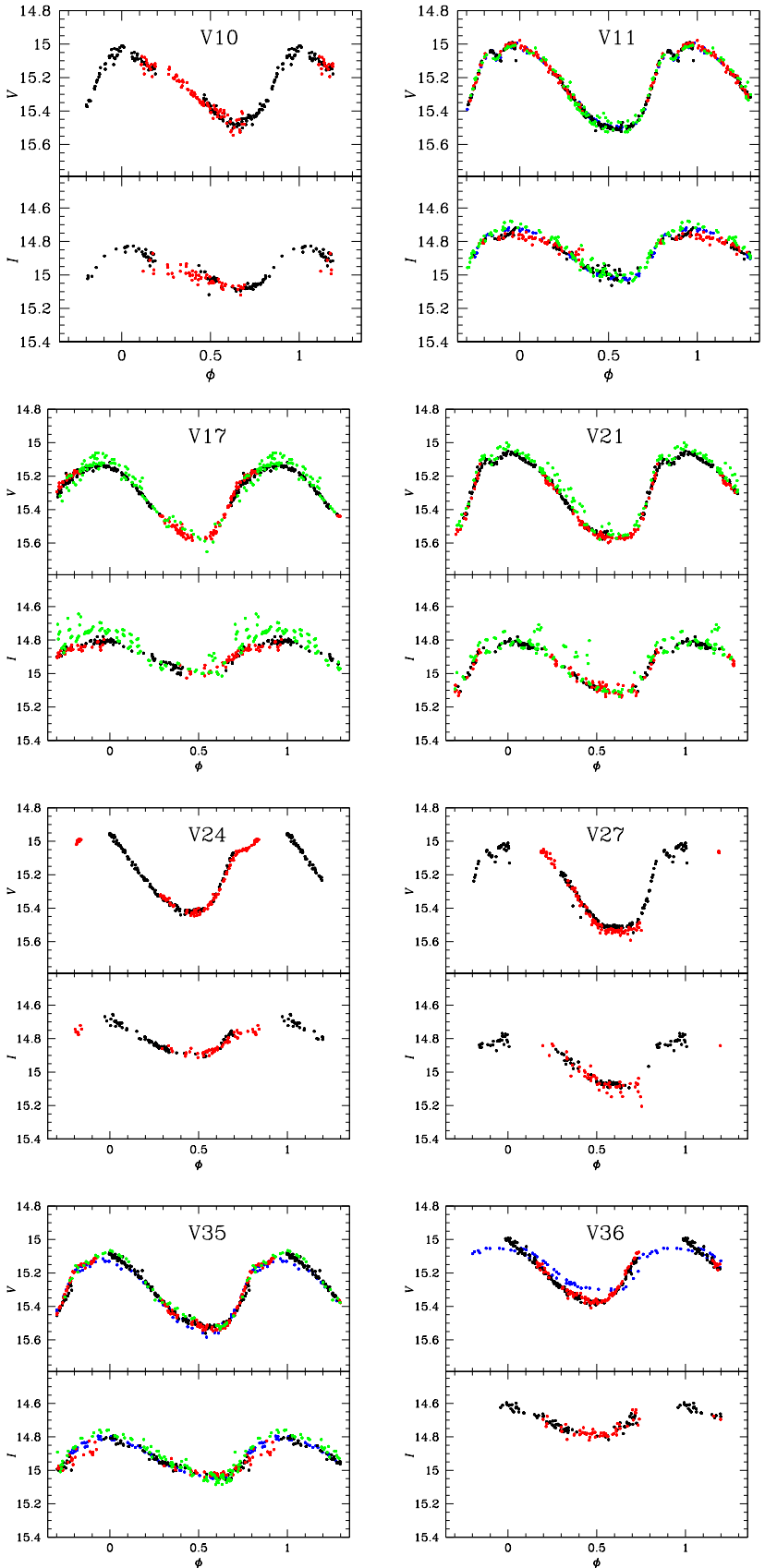

terminations from ESO/FLAMES spectra were obtained by Mucciarelli et al. (2016) and Massari et al. (2017) and obtained $[\mathrm{Fe} / \mathrm{H}]=-1.09 \pm 0.01$ and $-1.07 \pm 0.01$ respectively. Thus, our results are in very good agreement with recent spectroscopic estimations.

\subsection{The Color-Magnitude Diagram}

The color-magnitude diagram (CMD) in Fig. 4 was built using the magnitude weighted means of $V$ and $V-I$ for the 12245 stars in the field or our reference image from the run in Las Campanas. Variable stars are labelled and plotted 
Table 4 Fourier coefficients of RRab and RRc stars in NGC 6362. The numbers in parentheses indicate the uncertainty on the last decimal place. Also listed are the number of harmonics $N$ used to fit the light curve of each variable and the deviation parameter $D_{m}$.

\begin{tabular}{|c|c|c|c|c|c|c|c|c|c|c|}
\hline $\begin{array}{l}\text { Variable } \\
\text { ID }\end{array}$ & $\begin{array}{l}A_{0} \\
(V \text { mag })\end{array}$ & $\begin{array}{l}A_{1} \\
(V \text { mag })\end{array}$ & $\begin{array}{l}A_{2} \\
(V \text { mag })\end{array}$ & $\begin{array}{l}A_{3} \\
(V \text { mag })\end{array}$ & $\begin{array}{l}A_{4} \\
(V \text { mag })\end{array}$ & $\phi_{21}$ & $\phi_{31}$ & $\phi_{41}$ & $N$ & $D_{m}$ \\
\hline \multicolumn{11}{|c|}{ RRab } \\
\hline V2 & $15.368(2)$ & $0.4145(3)$ & $0.2088(3)$ & $0.1458(3)$ & $0.0974(3)$ & $3.888(2)$ & $8.147(3)$ & $6.034(4)$ & 10 & 1.7 \\
\hline V16 & $15.359(5)$ & $0.3442(3)$ & $0.1796(3)$ & $0.1212(3)$ & $0.0816(3)$ & $3.973(2)$ & $8.234(4)$ & $6.239(4)$ & 10 & 0.9 \\
\hline V19 & $15.365(2)$ & $0.2080(2)$ & $0.0945(2)$ & $0.0542(2)$ & $0.0235(2)$ & $4.172(3)$ & $8.656(4)$ & $7.097(9)$ & 10 & 1.0 \\
\hline V25 & $15.263(2)$ & $0.4195(4)$ & $0.2018(4)$ & $0.1510(4)$ & $0.0966(4)$ & $3.831(2)$ & $7.975(4)$ & $5.871(5)$ & 10 & 1.4 \\
\hline V26 & $15.351(1)$ & $0.2131(2)$ & $0.0973(2)$ & $0.0555(2)$ & $0.0245(2)$ & $4.200(3)$ & $8.690(5)$ & $7.139(9)$ & 10 & 1.3 \\
\hline \multicolumn{11}{|c|}{ RRc } \\
\hline V11 & $15.255(2)$ & $0.2434(2)$ & $0.0333(2)$ & $0.0207(2)$ & $0.0167(2)$ & $4.830(8)$ & $3.410(12)$ & $2.140(15)$ & 9 & \\
\hline V14 & $15.374(1)$ & $0.1587(4)$ & $0.0184(4)$ & $0.0040(4)$ & $0.0014(4)$ & $4.512(22)$ & $2.905(99)$ & $1.092(281)$ & 9 & \\
\hline V15 & $15.263(1)$ & $0.2192(2)$ & $0.0306(2)$ & $0.0190(2)$ & $0.0131(2)$ & $4.593(6)$ & $3.098(10)$ & $2.002(15)$ & 9 & \\
\hline V21 & $15.321(1)$ & $0.2366(2)$ & $0.0331(2)$ & $0.0222(2)$ & $0.0159(2)$ & $4.705(6)$ & $3.237(8)$ & $2.084(12)$ & 9 & \\
\hline V22 & $15.318(1)$ & $0.2303(8)$ & $0.0379(8)$ & $0.0148(8)$ & $0.0134(8)$ & $4.680(21)$ & $2.850(52)$ & $1.588(58)$ & 9 & \\
\hline V23 & $15.359(1)$ & $0.2428(2)$ & $0.0377(2)$ & $0.0232(2)$ & $0.0170(2)$ & $4.651(6)$ & $2.907(8)$ & $1.770(10)$ & 9 & \\
\hline
\end{tabular}

Table 5 Physical parameters of the stable RRab and RRc stars. The numbers in parentheses indicate the uncertainty on the last decimal places and have been calculated as described in the text.

\begin{tabular}{lccccccc}
\hline Star & {$[\mathrm{Fe} / \mathrm{H}]_{Z W}$} & {$[\mathrm{Fe} / \mathrm{H}]_{U V E S}$} & $M_{V}$ & $\log T_{\text {eff }}$ & $\log \left(L / \mathrm{L}_{\odot}\right)$ & $M / \mathrm{M}_{\odot}$ & $R / \mathrm{R}_{\odot}$ \\
\hline V2 & $-1.274(3)$ & $-1.157(3)$ & $0.633(1)$ & $3.821(7)$ & $1.647(1)$ & $0.69(6)$ & $5.09(1)$ \\
V16 & $-0.986(4)$ & $-0.887(4)$ & $0.789(1)$ & $3.826(7)$ & $1.584(1)$ & $0.65(5)$ & $4.63(1)$ \\
V19 & $-1.194(4)$ & $-1.075(3)$ & $0.637(1)$ & $3.806(7)$ & $1.645(1)$ & $0.62(5)$ & $5.46(1)$ \\
V25 & $-1.311(4)$ & $-1.195(4)$ & $0.688(1)$ & $3.823(7)$ & $1.625(1)$ & $0.71(6)$ & $4.93(1)$ \\
V26 & $-1.190(5)$ & $-1.211(32)$ & $0.610(3)$ & $3.824(10)$ & $1.656(1)$ & $0.51(6)$ & $5.09(1)$ \\
\hline Weighted mean & $-1.203(2)$ & $-1.066(2)$ & $0.657(1)$ & $3.816(3)$ & $1.637(1)$ & $0.66(2)$ & $5.22(1)$ \\
$\sigma$ & \pm 0.126 & \pm 0.126 & \pm 0.072 & \pm 0.007 & \pm 0.025 & \pm 0.07 & \pm 0.27 \\
\hline \multicolumn{7}{c}{ RRc } & \\
V11 & $-1.15(2)$ & $-1.03(2)$ & $0.566(1)$ & $3.872(1)$ & $1.674(1)$ & $0.56(1)$ & $4.16(1)$ \\
V14 & $-0.94(16)$ & $-0.85(11)$ & $0.683(2)$ & $3.878(1)$ & $1.627(1)$ & $0.68(2)$ & $3.84(4)$ \\
V15 & $-1.16(2)$ & $-1.11(2)$ & $0.601(1)$ & $3.872(1)$ & $1.660(1)$ & $0.60(1)$ & $4.09(1)$ \\
V21 & $-1.16(1)$ & $-1.04(1)$ & $0.582(1)$ & $3.873(1)$ & $1.667(1)$ & $0.58(1)$ & $4.11(1)$ \\
V22 & $-1.00(9)$ & $-0.90(6)$ & $0.621(4)$ & $3.877(1)$ & $1.652(1)$ & $0.62(4)$ & $3.96(2)$ \\
V23 & $-1.28(14)$ & $-1.16(1)$ & $0.585(1)$ & $3.872(1)$ & $1.666(1)$ & $0.59(1)$ & $4.12(1)$ \\
\hline Weighted mean & $-1.21(1)$ & $-1.08(1)$ & $0.589(1)$ & $3.872(1)$ & $1.664(1)$ & $0.59(1)$ & $4.10(1)$ \\
$\sigma$ & \pm 0.16 & \pm 0.16 & \pm 0.046 & \pm 0.003 & \pm 0.015 & \pm 0.04 & \pm 0.11 \\
\hline
\end{tabular}

using their intensity-weighted means $\langle V\rangle$ and $\langle V\rangle$ $-<I>$ calculated from the light curves in Figs. 2 and 3 and listed in Table 3 . All symbols and colours are explained in the caption of the figure. For the age of the cluster we adopted $12.1 \mathrm{Gyr}$ from the differential age determination of De Angeli et al. (2005). The isochrone and zahb model are from VandenBerg et al. (2014) and are shifted to the average distance modulus found from the RRL stars.

\subsection{Bailey diagram and Oosterhoff type}

The $\log \mathrm{P}$ vs Amplitude diagram, also known as the Bailey diagram, is a useful tool because it is a good discriminator between OoI and OoII clusters, the RRab and RRc stars are well separated and hence their classification is confirmed, and RRab stars of advanced evolution can be identified. Fig.
5 shows the diagram resulting from the data listed in Table 3 The full amplitudes were measured on the light curves of Figs. 2 and 3 , except in those cases were we missed the maximum or minimum. In these cases we estimated the full amplitude from the curves of Smo17. When amplitude modulations are evident, the maximum observed amplitude was taken. The distribution of the RR Lyrae stars in the diagram confirms NGC 6362 to be of the OoI type. Some outstanding stars deserve a comment. According to Cacciari et al. (2005), RRab stars closer to the dashed sequence are more evolved. V12, V13, V20 and V30 are therefore good candidates to be ahead in their evolution towards the AGB. These four stars have Blazhko modulations (Smo17). In the amplification of the HB star in Fig 7 they are among the most luminous RRab stars. Conversely the luminous V18 and V29 do not show signs of being more evolved. 

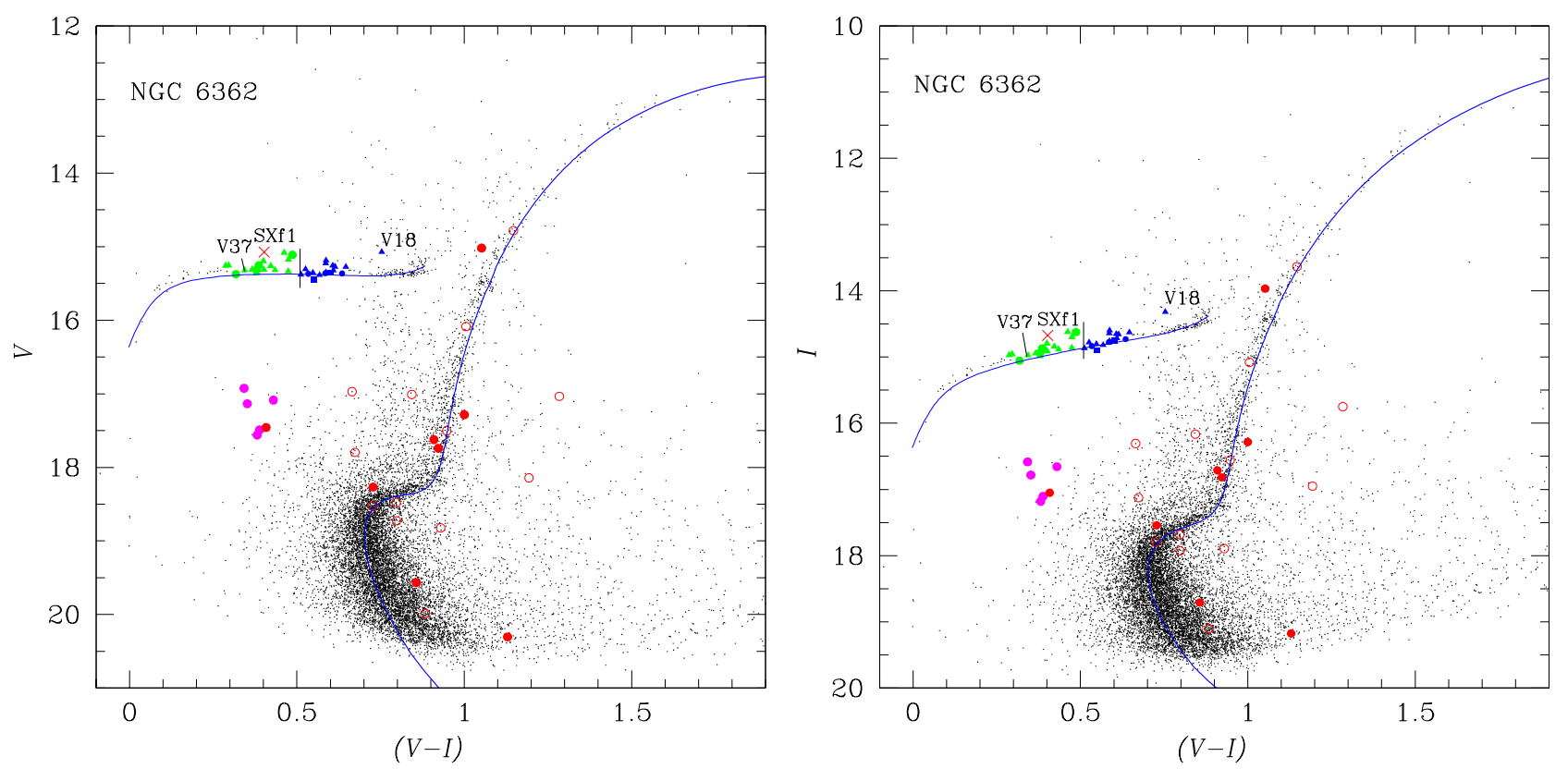

Fig. 4 CMD of NGC 6362. Coloured symbols are used for variable stars according to the following code: RRab (blue), RRc (green), SX Phe (purple), binaries in the field of the cluster discussed by Kałużny et al. (2014) that are members (filled red circles) and non-members (open red circles). The red cross shows the position of the newly found variable SXf1 of the SX Phe type. See appendix A for a discussion of the labelled stars. The isochrone for $12.1 \mathrm{Gyr},[\mathrm{Fe} / \mathrm{H}]=-1.0, \mathrm{Y}=0.25$ and $[\alpha / \mathrm{H}]=0.4$ and the slightly more metal poor zahb (-1.31) are from from VandenBerg et al. (2014), and have been reddened by $E(B-V)=0.063$ and shifted to the average distance modulus found from the RRL stars. The vertical black line shows the first overtone red edge calculated by Arellano Ferro et al. (2016) and shows the neat mode segregation.

\section{The SX Phoenicis stars in NGC 6362}

There are six SX Phe stars identified in NGC 6362. They are listed in Table 6 along with their intensity weighted magnitude, periods, pulsating mode, equatorial coordinates and the discovering paper. The pulsating modes were assigned from the position of the star on the log P- $V$ and the P-L relation of Cohen \& Sarajedini (2012) shifted to the predicted distance.

We have identified a new SX Phe in the field of our images in NGC 6362. In the CMD the star falls in an odd position for a member SX Phe (red cross in Fig. 4). The star is at least 2 magnitudes brighter than the SX Phe in the cluster, thus it must be a foreground object. Therefore, we refrain from assigning a sequential variable number to the star and identify it as SXf1. The light curves in our $V$ and $I$ photometry phased with a period of $0.07949 \mathrm{~d}$ is shown in Fig. A3 in appendix $\mathrm{A}$

\section{The Distance to NGC 6362}

The distance to NGC 6362 has been estimated from four independent approaches as described in the following sections.

\subsection{From the RR Lyrae stars}

To estimate the distance to NGC 6362 from the RR Lyrae stars we have converted the individual values of $M_{V}$ in Table 5 into individual distances and adopting $E(B-V)=0.063$ derived in $\S 3.1$ Since the values of $M_{V}$ come from independent calibrations from RRab and RRc stars we calculated two independent values of the true distance modulus; $14.499 \pm 0.078$ and $14.522 \pm 0.037$, corresponding to the distance $7.93 \pm 0.32$ and $8.02 \pm 0.15 \mathrm{kpc}$ from the RRab and the RRc stars respectively.

An independent estimate from I-band RR Lyrae P-L relation derived by Catelan et al. (2004) $M_{I}=0.471-$ $1.132 \log P+0.205 \log Z$, with $\log Z=[M / H]-1.765$. We applied these relations to the 34 RR Lyrae stars in Table 3. and found a distance of $7.85 \pm 0.37 \mathrm{kpc}$.

\subsection{From the SX Phe stars}

We have calculated the predicted distance to each SX Phe star, by the P-L relation of Cohen \& Sarajedini (2012), taking the periods and pulsating modes listed in Table 6 and assuming $E(B-V)=0.063$ for the six cluster members. The average distance is $8.07 \pm 0.44 \mathrm{kpc}$, in excellent agreement with distance from the RR Lyrae stars. In Fig 6 we show the distribution of SX Phe stars in the $\log \mathrm{P}-V$ plane, where the P-L relation of Cohen \& Sarajedini (2012) for the fundamental mode, shifted to the above distance, 
Table 6 SX Phe stars in the field of NGC 6362.

\begin{tabular}{lllllllll}
\hline $\begin{array}{l}\text { Variable } \\
\text { Star ID }\end{array}$ & $\begin{array}{l}<V> \\
(\mathrm{mag})\end{array}$ & $\begin{array}{l}<I> \\
(\mathrm{mag})\end{array}$ & $\begin{array}{l}P(\text { Kal14) } \\
(\mathrm{d})\end{array}$ & $\begin{array}{l}P \text { (this work) } \\
(\mathrm{d})\end{array}$ & $\begin{array}{l}\text { Pulsating } \\
\text { mode }\end{array}$ & $\begin{array}{l}\text { RA } \\
(\mathrm{J} 2000.0)\end{array}$ & $\begin{array}{l}\text { Dec } \\
(\mathrm{J} 2000.0)\end{array}$ & $\begin{array}{l}\text { Ref. } \\
\end{array}$ \\
& & & & & & & & \\
\hline V38 & 16.926 & 16.584 & 0.06661582 & 0.06661901 & $\mathrm{~F}$ & $17: 31: 43.7$ & $-67: 02: 58.0$ & 1 \\
V46 & 17.491 & 17.103 & 0.050634688 & 0.05063447 & $\mathrm{~F}$ & $17: 32: 25.0$ & $-67: 00: 31.4$ & 1 \\
V47 & 17.134 & 16.783 & 0.052234111 & 0.05223411 & $\mathrm{~F}$ & $17: 32: 13.0$ & $-67: 02: 38.1$ & 1 \\
V48 & 17.048 & - & 0.047920021 & 0.04656200 & 10 & $17: 31: 59.9$ & $-67: 03: 49.8$ & 1 \\
V64 & 17.086 & 16.656 & 0.050162402 & 0.05016240 & $1 \mathrm{O}$ & $17: 31: 58.2$ & $-67: 03: 45.8$ & 2 \\
V72 & 17.560 & 17.180 & 0.0436729 & 0.0436729 & $\mathrm{~F}$ & $17: 31: 29.0$ & $-67: 02: 33.9$ & 2 \\
SXf1 & 15.075 & 14.673 & - & 0.07949 & - & $17: 31: 09.8$ & $-66: 59: 35.6$ & 3 \\
\hline
\end{tabular}

1: Olech et al. (2001), 2 Kałużny et al. (2014), 3: Present work. Star is not a cluster member.

is displayed. The corresponding first overtone relation was placed assuming a first overtone to fundamental period ratio of P1/P0=0.783 (Santolamazza et al.|2001).

\subsection{Comments on the tip of the red giant branch method}

The luminosity of the true tip of the RGB can in principle be used to estimate the distance to globular clusters. The method, originally developed to estimate distances to nearby galaxies (Lee et al. 1993), has been used to corroborate the theoretical constrains that particle-physics may impose on the extension of the RGB in globular clusters, by comparing the predicted distance by the most luminous RGB with the results rendered by the RR Lyrae and SX Phe. Consistency between theoretical predictions and empirical calculations has been found by for NGC 6229 Arellano Ferro et al. (2015), for M5 Arellano Ferro et al. (2016) and for NGC 6934 Yepez et al. (2017). In brief, the bolometric absolute magnitude of the tip of the RGB was calibrated by Salaris \& Cassisi (1997) as:

$M_{b o l}^{t i p}=-3.949-0.178[M / H]+0.008[M / H]^{2}$,

where $[M / H]=[\mathrm{Fe} / \mathrm{H}]-\log (0.638 \mathrm{f}+0.362)$ and $\log \mathrm{f}=$ $[\alpha / \mathrm{Fe}]$ Salaris et al. (1993). Viaux et al. (2013) argued that the neutrino magnetic dipole moment enhances the plasma decay process, postpones helium ignition in low-mass stars, and extends the red giant branch in globular clusters. Hence, the true TRGB may be a bit brighter than the brightest observed stars by an amount between 0.04 and 0.16 mag.

For the case of NGC 6362, applying the largest correction of $0.16 \mathrm{mag}$, the method produces an absurdly large distance. We note that the separation between the Horizontal Branch (HB) and the brightest stars in the RGB in the above mentioned clusters, is about 3.3 and 4.0 mag in $V$ and I respectively, while in NGC 6362 it is only about 2.3 and $3.5 \mathrm{mag}$ (see also the CMD in Brocato et al. (1999)). The predicted $M_{b o l}^{\text {tip }}$ by eq. 6 for the metallicity and reddening of NGC 6362 is $-3.819 \mathrm{mag}$, and a correction of between 0.5 and $1.0 \mathrm{mag}$. would be necessary to bring the distance to about $8.0 \mathrm{kpc}$ as calculated from the RR Lyrae and SX Phe stars. Such correction is way out of the theoretical expectations. The method fails for NGC 6362. We do not really have an explanation for this but, it should be noted that the population of the RGB in this cluster is meager, that those stars at the tip of the RGB could rather be AGB stars, and that this is a much more metal rich cluster compared with those tested before.

\section{The horizontal branch of NGC 6362}

The neat splitting of RRc and RRab stars is clear in Fig. 7 This is significant given that, while the mode splitting has been found in all studied Oo II type clusters (nine so far) (Yepez et al. (2017)), it has been observed only some Oo I (three out of seven so far). Fig. 8, which is an updated version of Fig. 16 of Yepez et al. (2017), shows in the HB structure parameter $\mathcal{L}$ vs $[\mathrm{Fe} / \mathrm{H}]$ plane, the segregated clusters (black-rimmed symbols) and the non-segregated ones. NGC 6362 is now the third OoI cluster where we find the clean mode segregation, limited by the red edge of the first overtone instability strip. The other examples are NGC 6229 Arellano Ferro et al. (2015) and NGC 6171 (Deras et al. 2018 , in preparation). It has been discussed by Yepez et al. (2017) that the mode segregation versus the mingled mode distribution in the inter-mode or "either-or" region may be a consequence of the mass loss rates involved during the He flashes, and hence the resulting mass distribution on the ZAHB, which in turn controls the mode population of the inter-mode region (Caputo et al. 1978). It is remarkable that all metal-poor and older OoII type clusters do show the mode segregation.

\section{Summary}

In the present paper we publish new time-series CCD VI photometry of the variable stars in the globular cluster NGC 6362. Via the Fourier decomposition of the RR Lyrae stars we estimated the mean $[\mathrm{Fe} / \mathrm{H}]_{U V E S}$ as $-1.066 \pm 0.126$ and $-1.08 \pm 0.16$ and the distance $7.93 \pm 0.32$ and $8.02 \pm 0.15 \mathrm{kpc}$ from the RRab and RRc stars respectively. The reddening $E(B-V)=0.063 \pm 0.024$ was calculated using the intrinsic colour $(V-I)_{0}$ at minimum colour level for 15 RRab stars. These results agree very well with previous determinations found in the literature. 

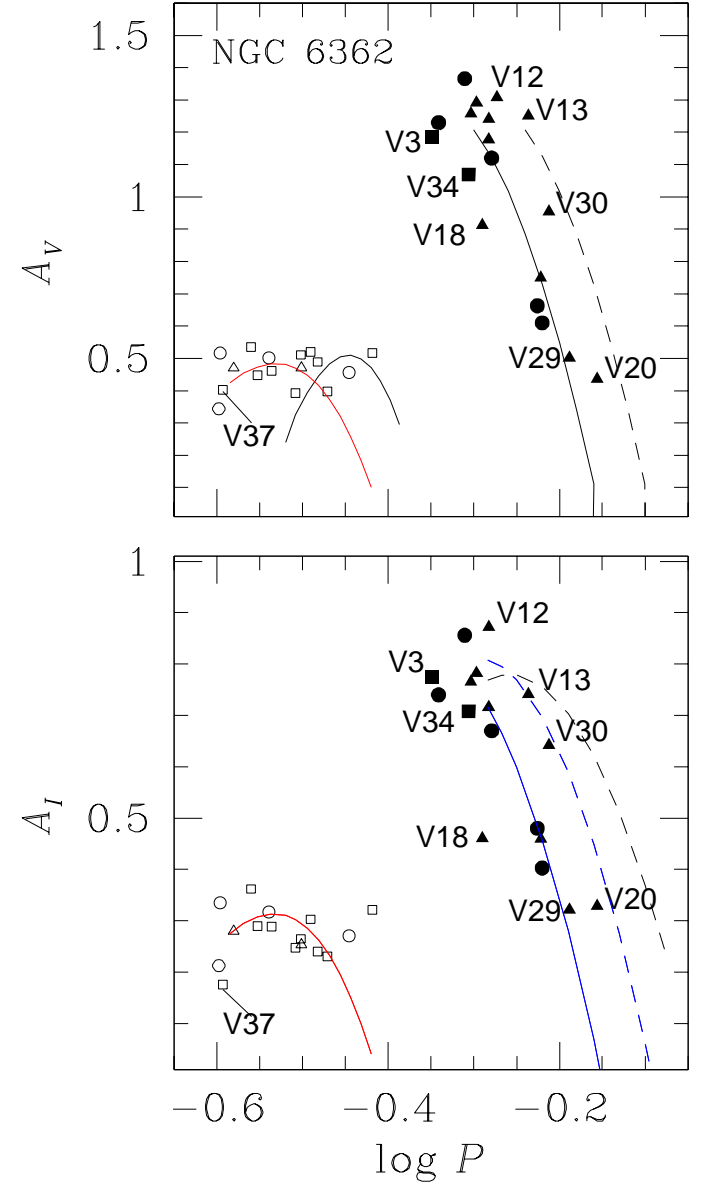

Fig.5 Period-Amplitude diagram for NGC 6362. Filled and open symbols represent RRab and RRc stars, respectively. Triangles and squares are used for stars with Blazhko modulations or double mode stars. The continuous and segmented lines in the top panel are the loci for evolved and unevolved stars in M3 according to Cacciari et al. (2005). The black parabola was obtained by Kunder et al. (2013a) from 14 OoII clusters. The red parabolas were calculated by Arellano Ferro et al. (2015) from a sample of RRc stars in five OoI clusters and avoiding Blazhko variables. In the bottom panel the black segmented locus was found by Arellano Ferro et al. (2011) and Arellano Ferro et al. (2013) for the OoII clusters NGC 5024 and NGC 6333 respectively. The blue loci are from Kunder et al. (2013b). See $\S 3.4$ for a discussion.

Our resulting CMD and the above parameters are in excelent agreement with models of the zero-age horizontal branch and the isochrone for $12.1 \mathrm{Gyr}$ from the VictoriaRegina stellar models of VandenBerg et al. (2014).

Employing the P-L relationship of Cohen \& Sarajedini (2012) for four fundamental mode and two first overtone SX Phe stars, an average distance of $8.07 \pm 0.44 \mathrm{kpc}$ was found, consistent with the results from the RR Lyrae stars.

We found a new SX Phe in the field of our images that seems to be a foreground star.

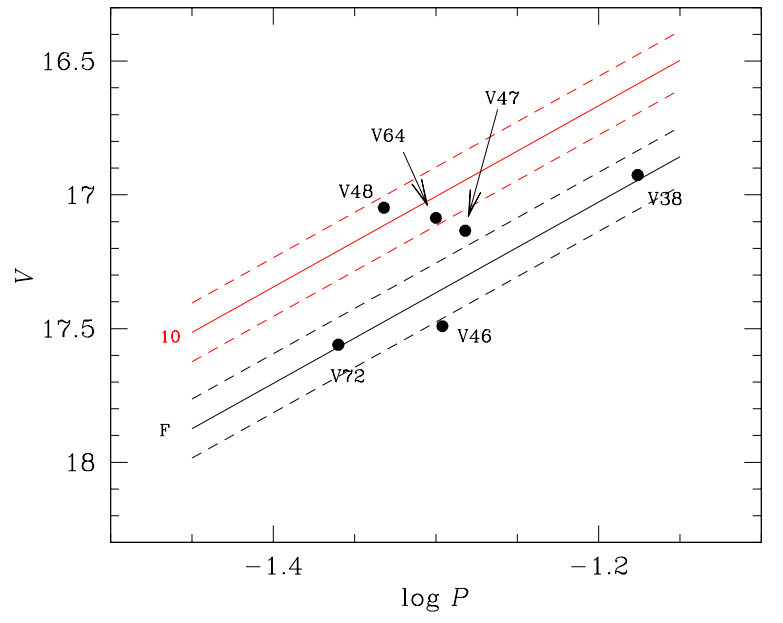

Fig. 6 The relation between period and luminosity for the SX Phe in NGC 6362. Black and red lines correspond to the P-L relation of Cohen \& Sarajedini (2012) for the fundamental and first overtone respectively, shifted to a distance of $8.07 \pm 0.44 \mathrm{kpc}$. Segmented lines correspond to zero-point uncertainty of the P-L relationship. The pulsating mode assignation to each star correspond to their position relative mode loci.

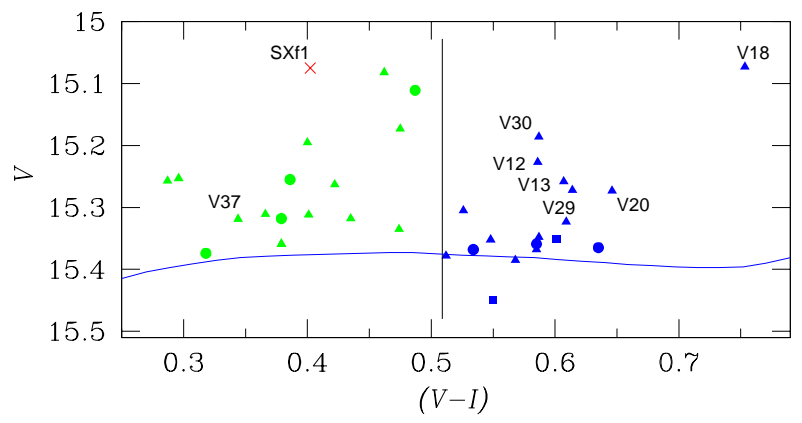

Fig. 7 The Horizontal Branch of NGC 6362. The stars $\mathrm{V} 12, \mathrm{~V} 13, \mathrm{~V} 20$ and V30 are among the most luminous of the RRab stars and their position in the the log P-Amplitude diagram (Fig. 5) suggests they are advanced in their evolution towards the AGB.

The distribution of the RRL stars in the HB and the Period-Amplitude diagram for this cluster reveal the presence of four RRab stars (V12, V13, V20 and V30) likely to be advanced in their evolution towards the AGB. If this is the case, as they evolve towards the red, they should display a positive secular period variation; a study yet to be performed. The fundamental and first overtone RRL stars, independently of whether they have Blazhko modulations and/or two excited pulsation modes, are neatly segregated around the red edge of the first overtone instability strip, which is a characteristic in OoII type clusters but only in some of the OoI clusters studied thus far.

Acknowledgements. We are grateful to Dr. Daniel Bramich for his DanDIA software and for guiding our data reduction process. AAF recognizes and thanks the support of UNAM via the DGAPA project IN104917. JAA wishes to thank the Instituto de 


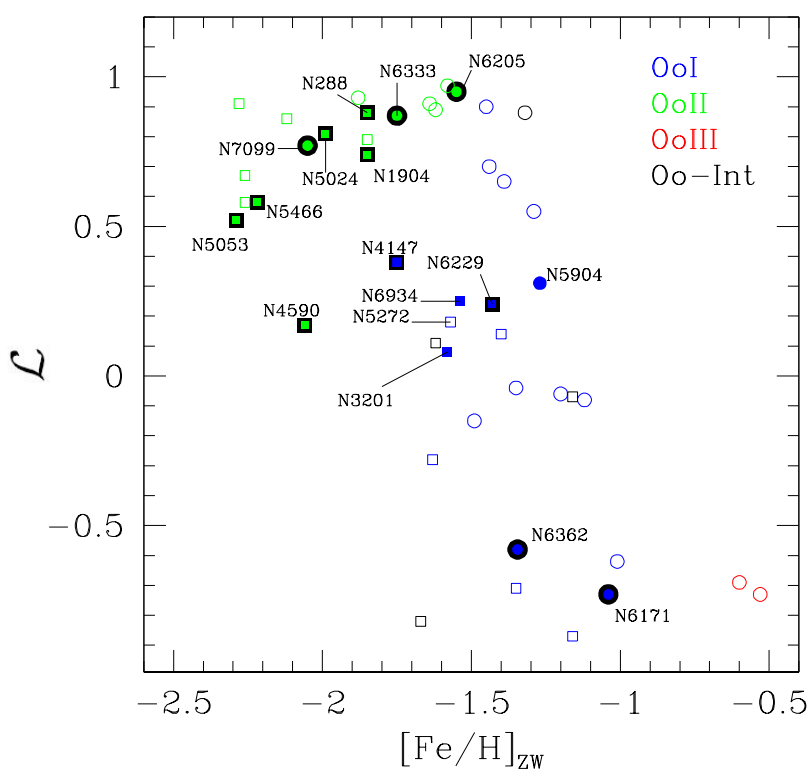

Fig. 8 The HB structure parameter $\mathcal{L}$ vs. metallicity. The black-rimmed symbols represent globular clusters where the fundamental and first overtone modes are well segregated around the first overtone red edge of the instability strip, as opposed to filled non-rimmed symbols. Empty symbols are clusters not studied by our group.

Astronomía of the Universidad Nacional Autónoma de Mexico for hospitality. We have made an extensive use of the SIMBAD and ADS services, for which we are thankful.

\section{References}

Arellano Ferro, A., Bramich, D. M., Figuera Jaimes, R., et al. 2013, MNRAS, 434, 1220

Arellano Ferro, A., Bramich, D. M., \& Giridhar, S. 2017, Rev. Mexicana Astron. Astrofis., 53, 121

Arellano Ferro, A., Figuera Jaimes, R., Giridhar, S., et al. 2011, MNRAS, 416, 2265

Arellano Ferro, A., Giridhar, S., \& Bramich, D. M. 2010, MNRAS, 402, 226

Arellano Ferro, A., Luna, A., Bramich, D. M., et al. 2016, Ap\&SS, 361,175

Arellano Ferro, A., Mancera Piña, P. E., Bramich, D. M., et al. 2015, MNRAS, 452, 727

Bramich, D. M. 2008, MNRAS, 386, L77

Bramich, D. M., Figuera Jaimes, R., Giridhar, S., \& Arellano Ferro, A. 2011, MNRAS, 413, 1275

Bramich, D. M. \& Freudling, W. 2012, MNRAS, 424, 1584

Bramich, D. M., Horne, K., Albrow, M. D., et al. 2013, MNRAS, 428, 2275

Brocato, E., Castellani, V., Raimondo, G., \& Walker, A. R. 1999, ApJ, 527, 230

Cacciari, C., Corwin, T. M., \& Carney, B. W. 2005, AJ, 129, 267

Carretta, E., Bragaglia, A., Gratton, R., D’Orazi, V., \& Lucatello, S. 2009, A\&A, 508, 695

Carretta, E. \& Gratton, R. G. 1997, A\&AS, 121, 95

Clement, C. M., Muzzin, A., Dufton, Q., et al. 2001, AJ, 122, 2587

Cohen, R. E. \& Sarajedini, A. 2012, MNRAS, 419, 342
Dalessandro, E., Massari, D., Bellazzini, M., et al. 2014, ApJ, 791, L4

De Angeli, F., Piotto, G., Cassisi, S., et al. 2005, AJ, 130, 116

Fourcade, C. R., Laborde, J. R., \& Albarracín, J. 1966, Atlas y Catálogo de Estrellas Variables en Cúmulos Globulares al sur de -29 grados. Universidad Nacional de Córdoba, Argentina

Guldenschuh, K. A., Layden, A. C., Wan, Y., et al. 2005, PASP, 117,721

Harris, W. E. 1996, AJ, 112, 1487

Jurcsik, J. 1998, A\&A, 333, 571

Jurcsik, J. \& Kovacs, G. 1996, A\&A, 312, 111

Kałużny, J., Thompson, I. B., Rozyczka, M., Pych, W., \& Narloch, W. 2014, Acta Astron., 64, 309

Kovács, G. 1998, Mem. Soc. Astron. Italiana, 69, 49

Kovacs, G. \& Kanbur, S. M. 1998, MNRAS, 295, 834

Kovács, G. \& Walker, A. R. 2001, A\&A, 374, 264

Kunder, A., Stetson, P. B., Cassisi, S., et al. 2013a, AJ, 146, 119

Kunder, A., Stetson, P. B., Catelan, M., Walker, A. R., \& Amigo, P. 2013b, AJ, 145, 33

Massari, D., Mucciarelli, A., Dalessandro, E., et al. 2017, MNRAS, 468, 1249

Mazur, B., Kałużny, J., \& Krzemiński, W. 1999, MNRAS, 306, 727

Morgan, S. M., Wahl, J. N., \& Wieckhorst, R. M. 2007, MNRAS, 374,1421

Mucciarelli, A., Dalessandro, E., Massari, D., et al. 2016, ApJ, 824,73

Olech, A., Kałużny, J., Thompson, I. B., et al. 2001, MNRAS, 321, 421

Oosterhoff, P. T. 1939, The Observatory, 62, 104

Piotto, G., Zoccali, M., King, I. R., et al. 1999, AJ, 117, 264

Ruciński, S. M. 2000, AJ, 120, 319

Rutledge, G. A., Hesser, J. E., \& Stetson, P. B. 1997, PASP, 109, 907

Salaris, M. \& Cassisi, S. 1997, MNRAS, 289, 406

Salaris, M., Chieffi, A., \& Straniero, O. 1993, ApJ, 414, 580

Samus, N. N., Kazarovets, E. V., Pastukhova, E. N., Tsvetkova, T. M., \& Durlevich, O. V. 2009, PASP, 121, 1378

Sandage, A. \& Cacciari, C. 1990, ApJ, 350, 645

Santolamazza, P., Marconi, M., Bono, G., et al. 2001, ApJ, 554, 1124

Sawyer Hogg, H. 1973, Publications of the David Dunlap Observatory, 3,1

Schlegel, D. J., Finkbeiner, D. P., \& Davis, M. 1998, ApJ, 500, 525

Stetson, P. B. 2000, PASP, 112, 925

Sturch, C. 1966, ApJ, 143, 774

van Agt, S. L. T. J. 1961, Bull. Astron. Inst. Netherlands, 15, 329

van Hoof, A. 1961, Louvain Pub., 14, 131

VandenBerg, D. A., Bergbusch, P. A., Ferguson, J. W., \& Edvardsson, B. 2014, ApJ, 794, 72

Viaux, N., Catelan, M., Stetson, P. B., et al. 2013, A\&A, 558, A12

Woods, I. E. \& Bailey, S. I. 1919, Harvard College Observatory Circular, 217, 1

Yepez, M. A., Arellano Ferro, A., Muneer, S., \& Giridhar, S. 2017, ArXiv e-prints 


\section{A Comments on individual variables}

\section{A.1 V18}

This star presents amplitude modulations and has been considered as a Blazhko variable by Olech et al. (2001) and Smo17. It appears as the most luminous and reddest RRab in the CMD of Fig. 4 The light curve in the $I$-band is of low amplitude and anomalously bright, hence the red position of the star, but no suggestion of advanced evolution is found from its position in the log P-Amplitude diagram.

\section{A.2 $\quad$ V37}

This variable was identified by Olech et al. (2001) as a double mode pulsator with one mode being non-radial. The star has been reconsidered by Smo17 and they identified the two modes, being at least one of them non-radial. On the basis of the light curve shape and amplitudes of the two modes, they postulate that the star is not a typical RRc but a beating variable of a new type. The light curve from our data is shown in Fig. A1 and, although less dense, it is similar and consistent with the light curves shown by Olech et al. (2001) and Smo17. We would like to note however, that the star is not isolated but that a very close neighbour is present at only 2.65 arc seconds to the SE from V37. In Fig. A2 we show this pair in the reference images of our CASLEO observations, of rather high seeing, and SWOPE with much better seeing conditions. Even under better conditions, in the SWOPE detector it is difficult to isolate and measure the two stars individually. Thus, contamination of V37 from the flux of the neighbour is most likely present in the photometry of previous and present studies. We have examined all differential images by blinking them along with the reference images and found that the star at the NW, i.e. that labelled V37, is variable and got some hint that star at the SE may also exhibit some variations, although this is not a firm conclusion given the blending conditions of the pair. If this proves to be true, then at least part of the observed modulations might be an artifact. A confirmation of the double mode nature and properties of V37 with a wider scale telescope is highly desired.

In the CMD (e.g. Fig. 7), V37 is near the hot edge of the first overtone instability strip although its colour has certainly been altered by the presence of the close neighbour. In the Log P Amplitude diagram it mingles well among the RRc stars and falls, as expected, near the predicted locus for the first overtones.

\section{A.3 SXf1}

The light curve of this star shows clear sinusoidal variations (Fig. A3 of small amplitude and a period of $0.07949 \mathrm{~d}$, typical of SX Phe stars. The star lies at the HB level, i.e. some 2 magnitudes brighter, although of similar colour, than the SX Phe cluster members. Therefore, we classify it as a foreground field SX Phe star.

\section{B Identification chart}

All the known variables in the field of our images are identified in charts B1 and B2 Kałużny et al. (2014) provided identification charts and RA and Dec coordinates, for all the variables stars in their study. We noted however that some coordinates did not correspond to their identification. M. Rozyczka (private comm.) pointed out that the coordinates of V42, V45, V49, V53-V58, V60, V63,

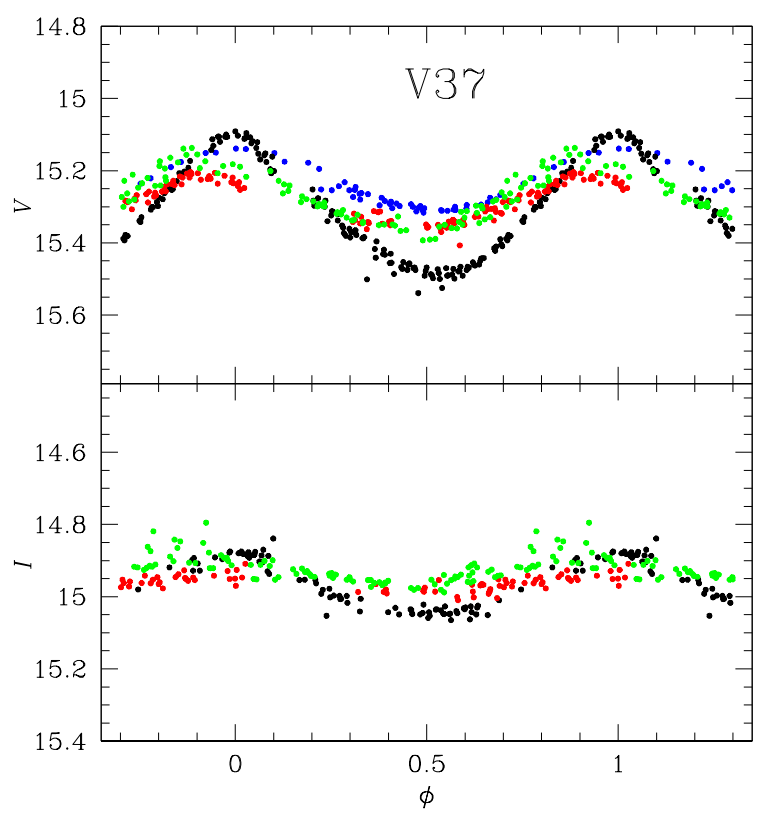

Fig. A1 V37 light curve. Colors are as in Fig. 2 Colours are as in Fig. 2 .
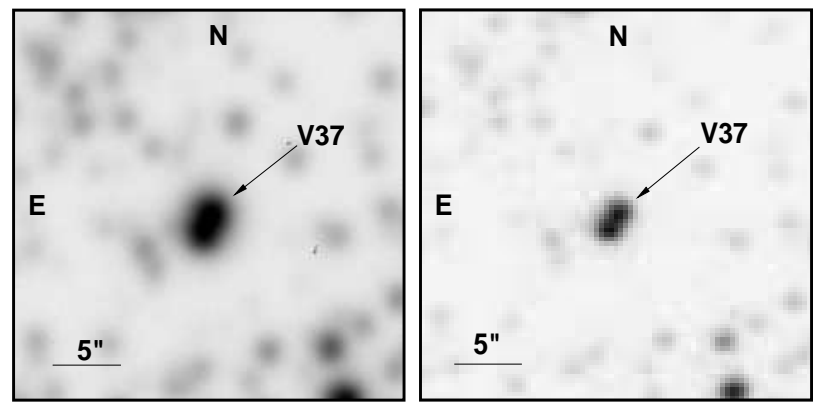

Fig. A2 V37 charts in the reference images from CASLEO (left) and from SWOPE (right).

and V75-V77 are in fact incorrect in their Table 1, and kindly provided the correct coordinates. Aiming to clarify the identifications of these stars, in Table B1 we list the correct coordinates, which are consistent with the identifications in the charts of Fig. B1 and B2 
Table B1 Corrected coordinates of binary stars in NGC 6362. The remainder of the binary stars are correctly identified in Table 1 of Kałużny et al. (2014).

\begin{tabular}{lllll}
\hline $\begin{array}{l}\text { Variable } \\
\text { Star ID }\end{array}$ & $\begin{array}{l}\text { RA } \\
\text { (decimal) }\end{array}$ & $\begin{array}{l}\text { DEC } \\
\text { (decimal) }\end{array}$ & $\begin{array}{l}\text { RA } \\
\text { (h m s) }\end{array}$ & $\begin{array}{l}\text { DEC } \\
\left({ }^{\prime}{ }^{\prime \prime}\right)\end{array}$ \\
\hline V42 & 262.78754 & -66.860809 & $17: 31: 09.0$ & $-66: 51: 38.9$ \\
V45 & 262.71952 & -66.983002 & $17: 30: 52.6$ & $-66: 58: 58.8$ \\
V49 & 263.10043 & -67.066788 & $17: 32: 24.1$ & $-67: 04: 00.4$ \\
V53 & 263.29060 & -66.856061 & $17: 33: 09.7$ & $-66: 51: 21.8$ \\
V54 & 263.22510 & -67.098769 & $17: 32: 54.0$ & $-67: 05: 55.5$ \\
V55 & 263.22533 & -66.926742 & $17: 32: 54.0$ & $-66: 55: 36.2$ \\
V56 & 263.21986 & -66.974452 & $17: 32: 52.7$ & $-66: 58: 28.0$ \\
V57 & 263.19214 & -66.925905 & $17: 32: 46.1$ & $-66: 55: 33.2$ \\
V58 & 263.11699 & -67.145429 & $17: 32: 28.0$ & $-67: 09: 43.5$ \\
V60 & 263.09729 & -66.924169 & $17: 32: 23: 3$ & $-66: 55: 27.0$ \\
V63 & 263.01446 & -67.139542 & $17: 32: 03.5$ & $-67: 08: 22.4$ \\
V75 & 262.80971 & -66.924412 & $17: 31: 14.3$ & $-66: 55: 27.8$ \\
V76 & 262.76813 & -67.056661 & $17: 31: 04.4$ & $-67: 03: 24: 0$ \\
V77 & 262.71325 & -66.924622 & $17: 30: 51.1$ & $-66: 55: 28.6$ \\
\hline
\end{tabular}

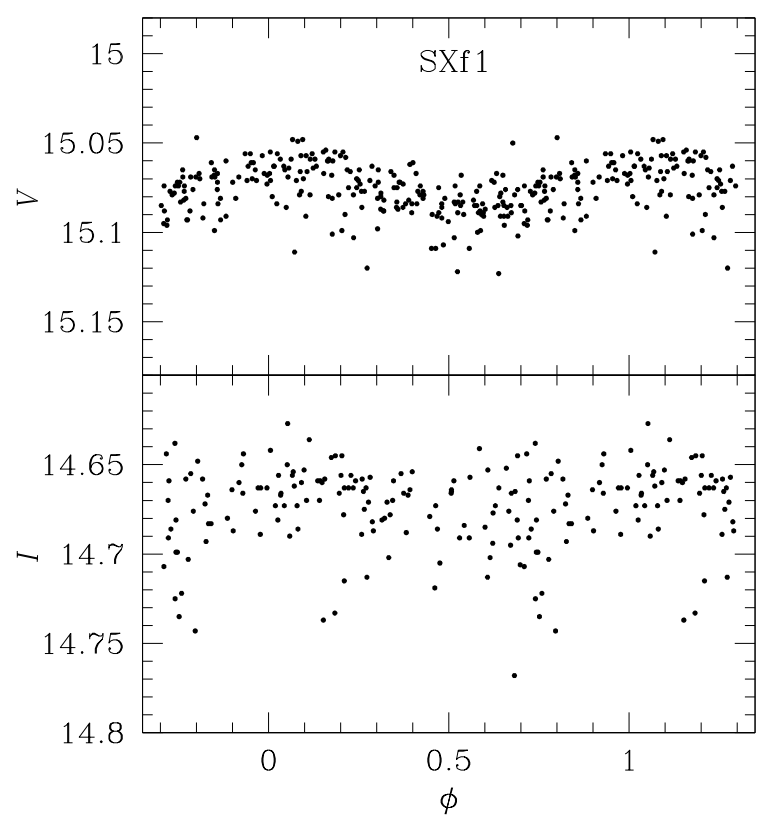

Fig. A3 The newly identified SX Phe in the field of NGC 6362. The star is not a cluster member but a foreground object. The light curves are phased with a period of 0.07949 d. 


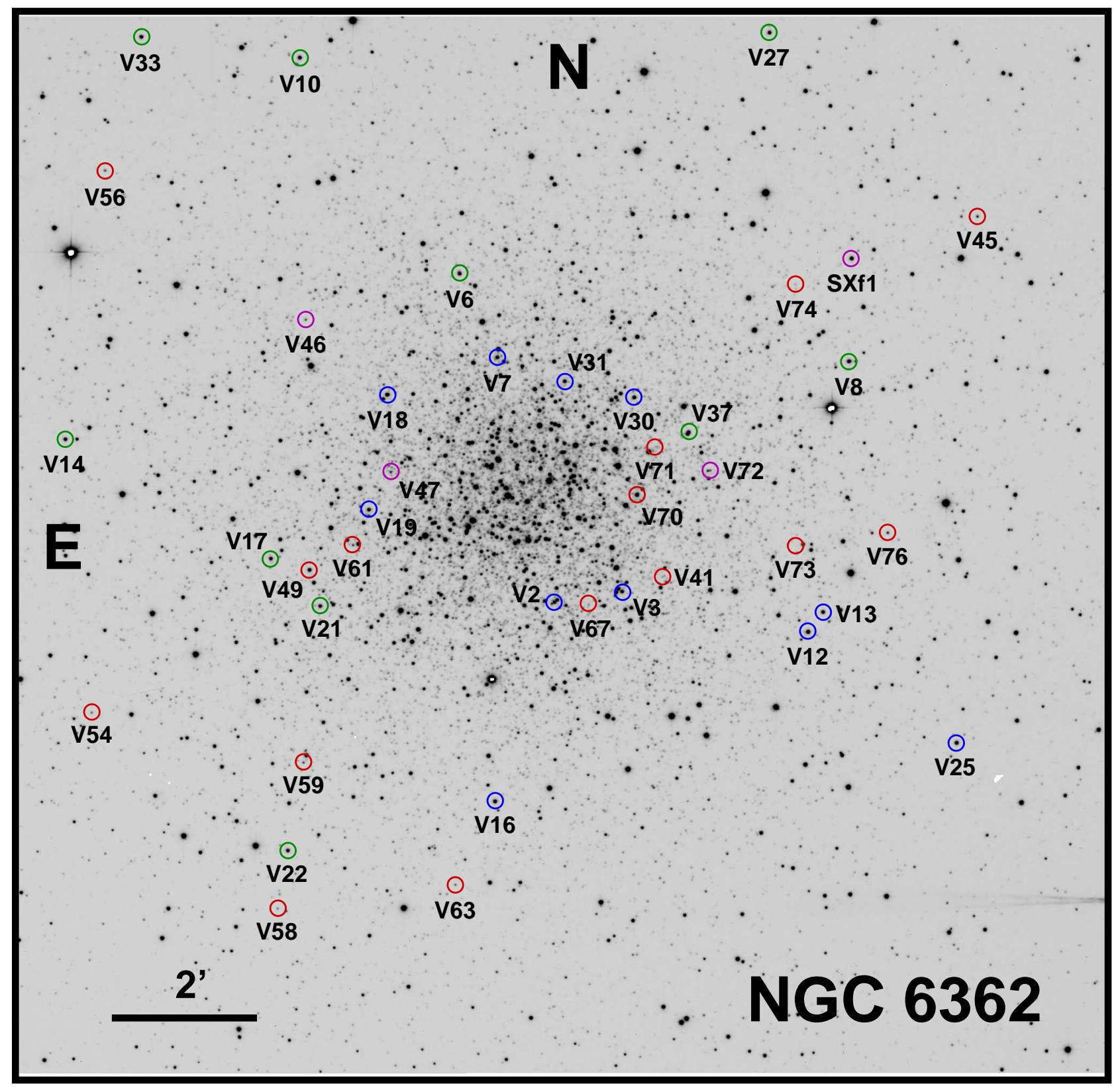

Fig. B1 Variable stars in the external field of NGC 6362. The image is the $V$ reference image employed for the differential imaging in the data from the SWOPE telescope. The field is about $14.5 \times 14.5 \mathrm{arcmin}^{2}$. The newly identified foreground SX Phe star is labelled as SXf1. Colours are used for different types of variables: blue for RRab stars, green for RRc, purple for SX Phe and red for eclipsing binaries. 


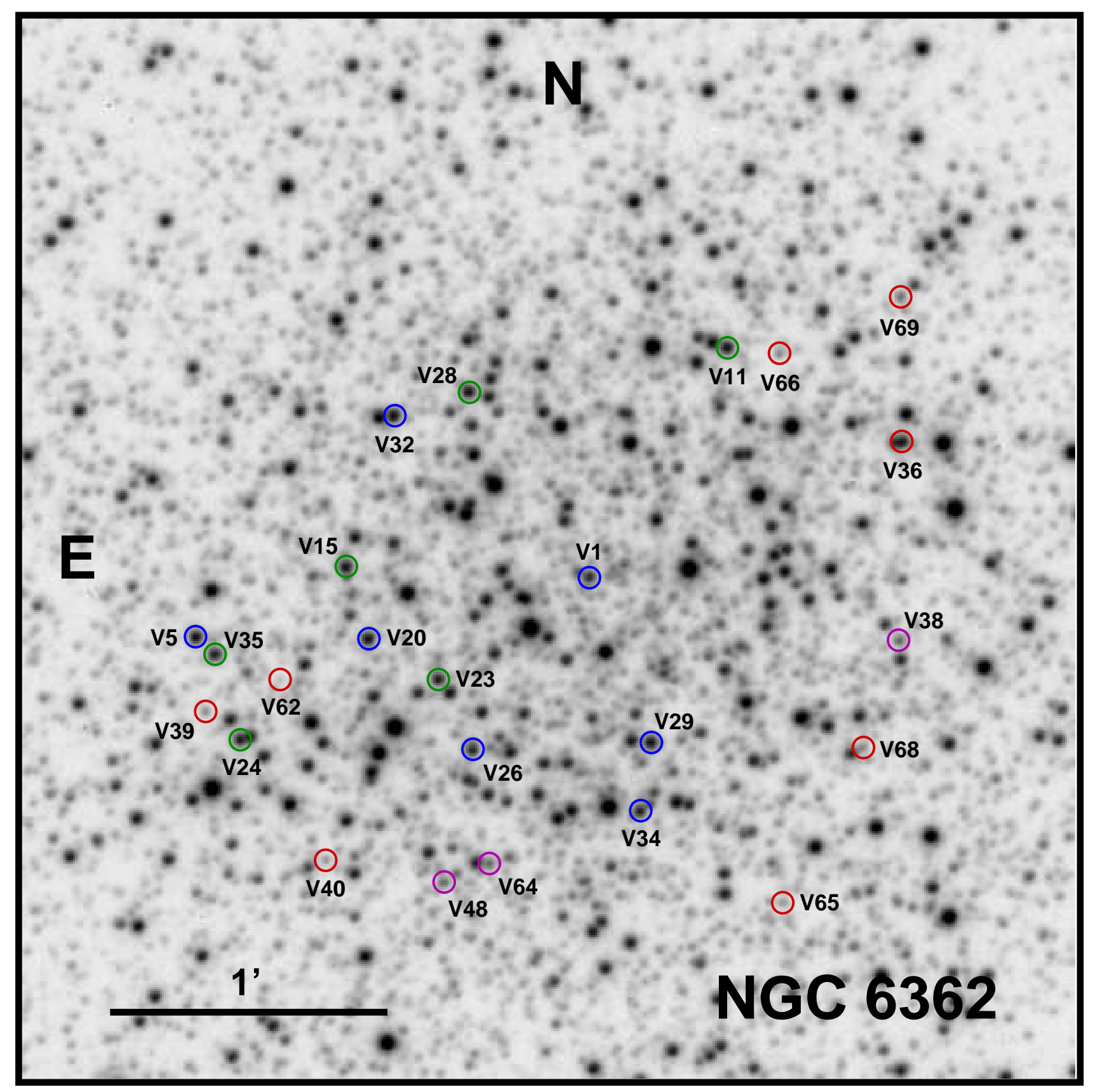

Fig. B2 Variable stars in the core region of NGC 6362. The field is about $3.75 \times 3.75 \mathrm{arcmin}^{2}$. The colour code is as in Fig. B1 